\title{
From the Gross-Pitaevskii equation to the Euler Korteweg system, existence of global strong solutions with small irrotational initial data
}

\author{
Corentin Audiard $^{1,2}$ (D) Boris Haspot ${ }^{3}$
}

Received: 2 May 2017 / Accepted: 30 September 2017 / Published online: 24 October 2017

(C) Fondazione Annali di Matematica Pura ed Applicata and Springer-Verlag GmbH Germany 2017

\begin{abstract}
We consider the Euler equations with a capillary tensor in the case of the so-called quantum hydrodynamics, which is formally equivalent to the Gross-Pitaevskii equation. Our main result is the existence and uniqueness of global solutions without vortices for small data in dimension at least 3 . The absence of vortices means that the density remains bounded away from 0 . Previous results include existence of global solutions without uniqueness (Antonelli and Marcati in Commun Math Phys 287(2):657-686, 2009) and lower bounds on the first occurrence of vortices (Béthuel et al. in J Anal Math 110:297-338, 2010). Our proof uses in a crucial way some deep results on the scattering of the Gross-Pitaevskii equation due to Gustafson et al. (Commun Contemp Math 11(4):657-707, 2009). The optimality of our assumptions is discussed, in particular we show that for slightly less regular initial data the density does not even remain bounded. We also sketch in the appendix the key arguments for the scattering of solutions of the Gross-Pitaevskii equation.
\end{abstract}

Keywords Fluid mechanics · Korteweg tensor - Global existence · Long-time dynamics · Scattering $\cdot$ Schrödinger type equations

Mathematics Subject Classification $35 \mathrm{Q} 35 \cdot 35 \mathrm{~A} 01 \cdot 35 \mathrm{~A} 02 \cdot 35 \mathrm{~B} 35 \cdot 35 \mathrm{~B} 40$

Corentin Audiard

corentin.audiard@1jll.math.upmc.fr

Boris Haspot

haspot@ceremade.dauphine.fr

1 UPMC Univ Paris 06, UMR 7598, Laboratoire Jacques-Louis Lions, Sorbonne Universités, 75005 Paris, France

2 CNRS, UMR 7598, Laboratoire Jacques-Louis Lions, 75005 Paris, France

3 Université Paris Dauphine, PSL Research University, Ceremade, UMR CNRS 7534, place du Maréchal De Lattre De Tassigny, 75775 Paris Cedex 16, France 


\section{Contents}

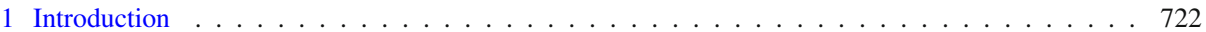

1.1 The Euler-Korteweg equations and the Madelung transform . . . . . . . . . . . . . . . . . 722

1.2 On the Gross-Pitaevskii equation . . . . . . . . . . . . . . . . . . . . . . . . . . . . . . . . 724

1.3 Main results . . . . . . . . . . . . . . . . . . . . . . . . . . . . . 727

Plan of the paper . . . . . . . . . . . . . . . . . . . . . . 728

2 Main tools . . . . . . . . . . . . . . . . . . . . . . . . . . . 729

2.1 Littlewood-Paley decomposition . . . . . . . . . . . . . . . . . . . . . . . . . . 729

2.2 Multilinear Fourier multipliers . . . . . . . . . . . . . . . . . . . . . . . . . 732

2.3 Strichartz and dispersive estimates . . . . . . . . . . . . . . . . . . . . . 733

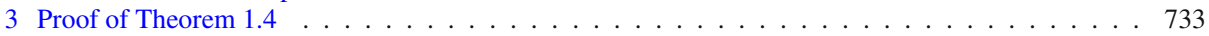

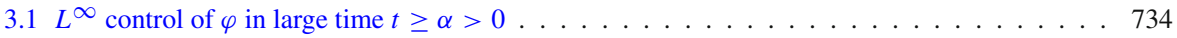

3.2 A smoothing property . . . . . . . . . . . . . . . . . . . . . . . 734

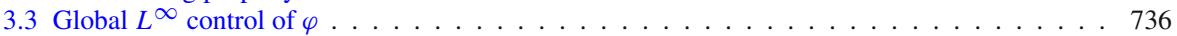

3.4 How to propagate the regularity from $\varphi$ to $\rho$ and $u \ldots \ldots \ldots \ldots \ldots$. . . . . . . . 737

3.5 Existence of global weak solution when $N=3 \ldots \ldots \ldots$. . . . . . . . . . . . . 739

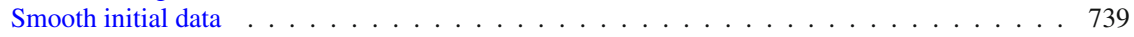

General case . . . . . . . . . . . . . . . . . . . . . . . . . 739

3.6 Existence of global strong solution when $N \geq 3 \ldots \ldots \ldots \ldots \ldots$. . . . . . . . . 740

Lipschitz control on the velocity $u \ldots \ldots \ldots \ldots$. . . . . . . . . . . . 746

4 Proof of Theorem 1.3: global well-posedness for $N \geq 4 \ldots \ldots$. . . . . . . . . . . 746

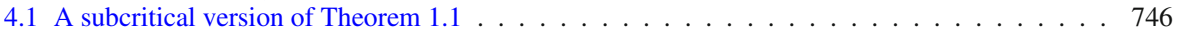

$4.2 L^{\infty}$ bounds . . . . . . . . . . . . . . . . . . . . . . . . . . . 748

Appendix A: Sketch of proof of Theorem 1.2 when $N=3 \ldots \ldots \ldots$. . . . . . . . . . . . 750

A.1 Space time resonances . . . . . . . . . . . . . . . . . . . . . . 750

A.2 Normal form . . . . . . . . . . . . . . . . . . . . . . . . 751

A.3 The functional space, reduction to a priori estimates . . . . . . . . . . . . . . 752

A.4 Control of the $S$ norm, action of the normal form . . . . . . . . . . . . . . . . . . 752

A.5 Control of the $X$ norm ． . . . . . . . . . . . . . . . . . . . . . . . . . . . 754

Appendix B: Some remarks on the sharpness . . . . . . . . . . . . . . . . . . . . . 758

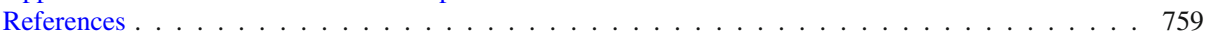

\section{Introduction}

\subsection{The Euler-Korteweg equations and the Madelung transform}

The motion of an Euler-Korteweg compressible fluid is described by the following system:

$$
\left\{\begin{array}{l}
\partial_{t} \rho+\operatorname{div}(\rho u)=0, \\
\partial_{t}(\rho u)+\operatorname{div}(\rho u \otimes u)+\nabla P(\rho)=\operatorname{div} K, \\
(\rho, u)_{t=0}=\left(\rho_{0}, u_{0}\right) .
\end{array}\right.
$$

Here $u=u(t, x) \in \mathbb{R}^{N}$ stands for the velocity field, $\rho=\rho(t, x) \in \mathbb{R}^{+}$is the density and $P$ the pressure. We restrict ourselves to the case $N \geq 3$. The general Korteweg tensor reads as follows:

$$
\operatorname{div} K=\operatorname{div}\left(\left(\rho \kappa(\rho) \Delta \rho+\frac{1}{2}\left(\kappa(\rho)+\rho \kappa^{\prime}(\rho)\right)|\nabla \rho|^{2}\right) I d-\kappa(\rho) \nabla \rho \otimes \nabla \rho\right) .
$$

The capillary coefficient $\kappa$ is a smooth function $\mathbb{R}^{+*} \rightarrow \mathbb{R}^{+*}$. The Euler-Korteweg system has been studied by Benzoni et al. in [5] where they prove for a general capillary coefficient the local existence of strong solutions for large data such that $\left(\rho_{0}-1, u_{0}\right)$ belong to $H^{s+1}\left(\mathbb{R}^{N}\right) \times$ $H^{s}\left(\mathbb{R}^{N}\right)$ with $s>\frac{N}{2}+1$. The proof relies on tricky energy inequalities, but the lack of uniform bounds does not allow to obtain global solutions. On the other hand, the equation has some 
dispersive structure so that global well-posedness is expectable at least if the dimension is large enough and the initial data small enough. Throughout the paper, we denote the space variable $x \in \mathbb{R}^{N}$ and we shall deal with the specific case:

$$
\kappa(\rho)=\frac{\kappa_{1}}{\rho} \quad \text { so that } \operatorname{div} K=2 \kappa_{1} \rho \nabla\left(\frac{\Delta \sqrt{\rho}}{\sqrt{\rho}}\right), \kappa_{1} \in \mathbb{R}^{+*}, P(\rho)=\rho^{2} / 2 .
$$

This capillary coefficient corresponds to the so-called quantum pressure. This case is of special interest because it corresponds to the fluid formulation of the Gross-Pitaevskii equation. More precisely, when the velocity $u=\nabla \theta$ is irrotational, $\lim _{|x| \rightarrow+\infty} \rho=1$, the Madelung transform $\psi=\sqrt{\rho} e^{i \frac{\theta}{2 \sqrt{k_{1}}}}$ allows formally to rewrite the Euler-Korteweg system as the Gross-Pitaevski equation ${ }^{1}$ (GP):

$$
\left\{\begin{array}{l}
2 i \sqrt{\kappa_{1}} \partial_{t} \psi+2 \kappa_{1} \Delta \psi=\left(|\psi|^{2}-1\right) \psi \\
\psi(0, \cdot)=\psi_{0}
\end{array}\right.
$$

with the boundary condition $\lim _{|x| \rightarrow+\infty}|\psi|=1$. The Gross-Pitaevskii equation is the Hamiltonian evolution associated to the Ginzburg-Landau energy:

$$
\begin{aligned}
E(\psi) & =\int_{\mathbb{R}^{N}}\left(\kappa_{1}|\nabla \psi(t, x)|^{2}+\frac{1}{4}\left(|\psi|^{2}-1\right)^{2}\right) d x \\
& =\int_{\mathbb{R}^{N}}\left(\kappa_{1}|\nabla \varphi(t, x)|^{2}+\frac{1}{4}\left(2 \operatorname{Re} \varphi+|\varphi|^{2}\right)^{2}\right) d x .
\end{aligned}
$$

with $\psi=1+\varphi$. Note that the fluid counterpart of this energy can be obtained by multiplying the momentum equation by $2 u$ and reads

$$
\begin{aligned}
& \int_{\mathbb{R}^{N}}\left(4 \kappa_{1}|\nabla \sqrt{\rho}|^{2}(t, x)+\left(\rho|u|^{2}\right)(t, x)+(\rho-1)^{2}(t, x)\right) d x \\
& =\int_{\mathbb{R}^{N}}\left(4 \kappa_{1}\left|\nabla \sqrt{\rho_{0}}\right|^{2}(x)+\left(\rho_{0}\left|u_{0}\right|^{2}\right)(x)+\left(\rho_{0}-1\right)^{2}(x)\right) d x .
\end{aligned}
$$

Taking advantage of this correspondence, Antonelli and Marcati proved in [1,2] the existence of global weak solution for the system (1.1) for irrotational initial data when $N=2,3$ and for pressures that correspond to defocusing nonlinear Schrödinger equations (NLS) (see also [11] for a simpler argument). It is important to mention that in $[1,2]$ the authors deal with initial density which are close to the vacuum, indeed $\rho_{0}$ belongs to $L^{2}\left(\mathbb{R}^{N}\right)$. The proofs consist in constructing a sequence of global smooth solutions of the system (1.1) (for regularized initial data). The main difficulty to pass from a solution of NLS to a solution of (1.1) is that $\psi$ can vanish, so that $u=\operatorname{Im}\left(\bar{\psi} \nabla \psi /|\psi|^{2}\right)$ is not clearly defined, even as a distribution. Next they prove the convergence to a global weak solution of the system (1.1). The key point of the proof is the strong $L_{\text {loc }}^{2}$ convergence of the nonlinear terms $\sqrt{\rho_{n}} u_{n} \otimes \sqrt{\rho_{n}} u_{n}$ and $\left|\nabla \sqrt{\rho_{n}}\right|^{2}$. This terms are in fact intertwined and converge using classical regularizing effects of Kato type for the Schrödinger equation. Uniqueness was left open as no control of the vacuum was provided.

On the other hand, Béthuel et al. studied in [6] the Gross-Pitaevskii equation in the long wave regime (small data and slow oscillations), and proved the well-posedness of (1.1) for large times. More precisely, they prove that for such times the density remains bounded away

\footnotetext{
${ }^{1}$ It should be pointed out that for a general capillarity the Euler-Korteweg system can also be rewritten as some degenerate quasi-linear Schrödinger equation, see [5]. The change of variable does not involve the Madelung transform but is still singular near vacuum.
} 
from zero, which in this context corresponds to the absence of vortices. It relied in a crucial way on dispersive properties of the Schrödinger equation. However, the question of global well-posedness was left open.

The main novelty of our results is that we construct solutions that are both unique, without vortices, and global. The price to pay is that we need to take small initial data and the dimension $N \geq 3$ in order to fully benefit of dispersive effects. Let us mention that we obtained very recently in [3] the global well-posedness of (1.1) for small initial data and general capillarity and pressure by a direct approach. In particular the Madelung transform is not used. The drawback is that much stronger restrictions on the regularity of the initial data are required (basically $\rho_{0} \in 1+H^{50}$ ).

In this work we loosen the assumptions on the initial data : first we build upon the scattering results for (GP) (see [27-29]) to construct a global solution $\psi$ that remains bounded away from 0 but is merely in $L^{\infty} \cap H^{s}$ with $s \simeq N / 2$, second we use the Madelung transform (which is well-defined since $\psi$ is bounded away from 0 ) to construct a global strong solution of the system (1.1). Uniqueness requires $\left(\rho_{0}, u_{0}\right) \in\left(1+H^{s+1}\right) \times H^{s}, s>N / 2+1$.

Before stating our main results we give a (incomplete) review on scattering for NLS and Gross-Pitaevskii.

\subsection{On the Gross-Pitaevskii equation}

Global well-posedness and solitons: Due to the unusual boundary condition at infinity, the analysis of the Cauchy problem for the Gross-Pitaevskii equation is more involved than for a defocusing NLS. Up to a change of variable and for simplicity we can replace (1.3) by the normalized PDE $i \partial_{t} \psi+\Delta \psi=\left(|\psi|^{2}-1\right) \psi$. The natural energy space is not $H^{1}\left(\mathbb{R}^{N}\right)$, and the $L^{2}\left(\mathbb{R}^{N}\right)$ norm is not conserved (we will see that it is related to the low frequencies behavior of the linearized equation near $\psi=1$ ). The natural energy space associated to the Gross-Pitaevskii equation is

$$
E_{1}=\left\{\psi \in H_{l o c}^{1}\left(\mathbb{R}^{N}\right), \nabla \psi \in L^{2}\left(\mathbb{R}^{N}\right),|\psi|^{2}-1 \in L^{2}\left(\mathbb{R}^{N}\right)\right\} .
$$

Global well-posedness with large initial data in $E_{1}$ has been proved by Gallo and Gérard in $[17,18]$ in dimension $N \leq 3$ and by Kilipp et al. in [35] in the critical case $N=4$. It was also proved that for $s \geq 1$ the $H^{s}\left(\mathbb{R}^{N}\right)$ regularity is also propagated but without uniform bounds in time.

A striking difference between (GP) and defocusing Schrödinger equations is the existence of traveling waves, namely solutions of the form (up to symmetry):

$$
\psi(t, x)=u_{c}\left(x_{1}-c t, x_{2}, \ldots, x_{N}\right),
$$

where $u_{c}$ satisfies:

$$
i c \partial_{1} u_{c}-\Delta u_{c}-u_{c}\left(1-\left|u_{c}\right|^{2}\right)=0 .
$$

For $N \geq 2$, due to the correspondence with the Euler equations, it was conjectured more than thirty years ago that non-constant solutions do not exist for $|c|>\sqrt{2}$, this was rigorously proved in [24]). Solutions of finite energy were constructed for small $c$ in the pioneering paper [8], and the full range $0<|c|<\sqrt{2}$ was obtained by Maris in [36] in dimension $N \geq 3$. Béthuel et al. proved in [7] that there is a lower bound on the energy of non-trivial traveling waves for (1.8) in dimension $N=3$, the result was then extended in any dimension $\geq 4$ by de Laire [16] 


$$
0<\mathcal{E}_{0}=\inf \left\{E(\psi), \psi(t, x)=u_{c}\left(x_{1}-c t, x_{2}, \ldots, x_{N}\right) \text { solves }(1.8) \text { for } c>0\right\} .
$$

On the other hand if $N=2$ there exist non-trivial traveling waves of arbitrary small energy (this it was conjectured by Jones et al. [33], see [7] for a proof). This is a clear obstruction to scattering.

The scattering problem: We rewrite (1.3) for $\varphi=\psi-1$ :

$$
i \partial_{t} \varphi+\Delta \varphi-2 \operatorname{Re} \varphi=F(\varphi)=\left(\varphi+2 \bar{\varphi}+|\varphi|^{2}\right) \varphi .
$$

The strongest nonlinearity $|\varphi|^{2} \varphi$ corresponds to the defocusing cubic nonlinear Schrödinger equation, but the dynamic is actually very different. The linearized system reads

$$
i \partial_{t} \varphi+\Delta \varphi-2 \operatorname{Re} \varphi=0 .
$$

The system on $\operatorname{Re}(\varphi), \operatorname{Im}(\varphi)$ can be diagonalized by the change of unknown (see [27])

$$
v_{1}+i v_{2}=v:=V \varphi:=\operatorname{Re} \varphi+i U \operatorname{Im} \varphi \text { with } U=\sqrt{-\Delta(2-\Delta)^{-1}},
$$

and setting $H=\sqrt{-\Delta(2-\Delta)}$ we get the linear Schrödinger-like equation:

$$
i \partial_{t} v-H v=0 .
$$

For completeness we recall what we mean by "scattering". Consider the nonlinear Schrödinger-like equation:

$$
\left\{\begin{array}{l}
i \partial_{t} u+A(-\Delta) u=f(u), \\
u(0)=u_{0},
\end{array}\right.
$$

where $A(-\Delta)$ is a Fourier multiplier with real valued symbol. A solution is said to scatter (in $\left.L^{2}\right)$ if it is global, $C\left(\mathbb{R}^{+}, L^{2}\right)$, and there is $u_{+} \in L^{2}$ such that $\left\|e^{-i t A} u(t)-u_{+}\right\|_{L^{2}} \rightarrow_{t \rightarrow+\infty} 0$. This should be understood as a domination of the dispersive decay over nonlinear effects.

A very natural framework for scattering corresponds to the case where the equation has an energy and is globally well-posed in the energy space. The case of defocusing subcritical Schrödinger equations is relatively well understood even for large initial data (e.g., [12] chapter 7). Let us mention that over the last 15 years spectacular progress was made for critical Schrödinger equation : following the groundbreaking results in [15] where scattering was obtained for the quintic NLS in dimension 3, an extremely abondant literature has developed, for example the global well-posedness and scattering for the critical defocusing cubic Schrödinger equation $(N=4)$ was proved in [37].

However, despite the existence of a positive energy for Gross-Pitaevskii, the analogy with defocusing NLS should not be overestimated, as for the former solitons exist and even for small data scattering in dimension 2 is not expected to be true. Actually, the scattering problem for $(G P)$ is more similar to the following quadratic NLS:

$$
i \partial_{t} \varphi+\Delta \varphi=\lambda \varphi^{2},\left.\varphi\right|_{t=0}=\varphi_{0} .
$$

The Duhamel formula reads $\varphi(t)=e^{i t \Delta} \varphi_{0}-i \int_{0}^{t} e^{i(t-s) \Delta} f(\varphi(s)) d s$. Due to dispersive decay, $\left\|e^{i t \Delta} \varphi_{0}\right\|_{L^{3}} \lesssim\left\|\varphi_{0}\right\|_{L^{3 / 2} / t^{N / 6}}$. Assume that $\|\varphi(t)\|_{L^{3}} \leq \varepsilon / t^{N / 6}$, then the dispersion gives in the Duhamel part

$$
\begin{aligned}
\left\|\int_{1}^{t} e^{i(t-s) \Delta} \varphi^{2}(s) d s\right\|_{L^{3}} & \lesssim \int_{1}^{t} \frac{\|\varphi(s)\|_{L^{3}}^{2}}{(t-s)^{N / 6}} d s \\
& \leq \int_{1}^{t} \frac{\varepsilon^{2}}{(t-s)^{N / 6} s} d s
\end{aligned}
$$




$$
\lesssim\left\{\begin{array}{l}
\frac{\varepsilon^{2} \ln t}{\sqrt{t}}, N=3 \\
\frac{C \varepsilon^{2}}{t^{N / 6}}, N \geq 4 .
\end{array}\right.
$$

so that the direct approach fails to get closed estimates in dimension 3 , but any exponent in the nonlinearity larger than 2 or dimension larger than 3 can be handled. The general case was treated by Strauss [38] who proved that if the nonlinearity is basically a power $\alpha>\alpha_{0}$ with

$$
\alpha_{0}(N)=\frac{2+N+\sqrt{N^{2}+12 N+4}}{2 N} .
$$

then global well-posedness holds for small initial data. This does not mean that solutions of quadratic NLS in dimension 3 cannot scatter, but rather that the structure of the nonlinearity matters. The case of nonlinearities of the type $\lambda_{1} u^{2}+\lambda_{2} \bar{u}^{2}$ was treated by Hayashi and Naumkin [31], Hayashi et al. [30], Kawahara [34]. For the nonlinearity $|u|^{2}$, almost global existence has been proved by Ginibre and Hayashi [22], but there is no result of global existence. As a way to clarify the structure of nonlinearities (and handle them), the notion of space-time resonance has been introduced independently by Germain et al. in [19] and Gustafson et al. in [29].

In the case of Gross-Pitaevskii, diagonalized (1.8) reads:

$$
\begin{aligned}
i \partial_{t} v-H v= & U\left(3 v_{1}^{2}+\left(U^{-1} v_{2}\right)^{2}+\left|v_{1}+i U^{-1} v_{2}\right|^{2} v_{1}\right) \\
& +i\left(2 v_{1}\left(U^{-1} v_{2}\right)+\left|v_{1}+i U^{-1} v_{2}\right|^{2}\left(U^{-1} v_{2}\right)\right),
\end{aligned}
$$

which seems extremely bad as (1.12) contains quadratic nonlinearities of type $|v|^{2}$ with singular factors $U^{-1} v_{2}$. Nevertheless for $N \geq 3$, scattering for $(G P)$ has been obtained in a series of recent papers by Gustafson et al. [27-29]. They involve two key arguments: a normal form transform to "desingularize" the nonlinearity, and in the difficult case $N=3$ a subtle analysis of the space-time resonances. The case $N=3$ requires the data to be small in weighted $H^{1}\left(\mathbb{R}^{N}\right)$ spaces (to which, nevertheless, traveling waves belong, see [25] for the decay rate in space of the traveling waves). For the convenience of the reader we include in Appendix A a short description of the normal form transform and the method of space-time resonances developed in [29]. The main results of [27,29]read as follows:

Theorem 1.1 (Gustafson et al. [27]). Suppose that $N \geq 4$ and $|\sigma| \leq \frac{N-3}{2}-\frac{1}{N}, U^{\sigma} U^{-1} V \varphi_{0}$ is sufficiently small in $H^{\frac{N}{2}-1}\left(\mathbb{R}^{N}\right)$, then $U^{\sigma} U^{-1} V \varphi(t)$ remains small in $H^{\frac{N}{2}-1}\left(\mathbb{R}^{N}\right)$ for all $t \in \mathbb{R}$. Moreover, there exist $v_{ \pm} \in U^{-\sigma} H^{\frac{N}{2}-1}\left(\mathbb{R}^{N}\right)$ such that:

$$
\left\|U^{\sigma}\left(e^{i t H} U^{-1} V \varphi(t)-v_{ \pm}\right)\right\|_{H^{\frac{N}{2}-1}\left(\mathbb{R}^{N}\right)} \longrightarrow \pm \infty 0,
$$

and the wave operators $v_{ \pm} \rightarrow U^{-1} V u(0)$ are local homeomorphisms near 0 in $U^{-\sigma} H^{\frac{N}{2}-1}\left(\mathbb{R}^{N}\right)$.

Below we denote $\langle x\rangle=\sqrt{2+|x|^{2}}$ and $\langle x\rangle^{-1} H^{1}$ is the weighted space with norm $\|\langle x\rangle v\|_{H^{1}}$ and $\langle\nabla\rangle=\sqrt{2-\Delta}$.In dimension $N=3$ for small initial data, Gustafson, Nakanishi, Tsai obtain the following result.

Theorem 1.2 (Gustafson et al. [29]). Let $N=3$. There exists $\delta>0$ such that for any $\varphi_{0} \in H^{1}\left(\mathbb{R}^{3}\right)$ satisfying:

$$
\int_{\mathbb{R}^{3}}\langle x\rangle^{2}\left(\left|\operatorname{Re}\left(\varphi_{0}\right)\right|^{2}+\left|\nabla \varphi_{0}\right|^{2}\right)<\delta,
$$


then there exists a unique global strong solution $\psi=1+\varphi$ of (1.8) such that $v=V \varphi=$ $\operatorname{Re} \varphi+i U \operatorname{Im} \varphi$ satisfies $e^{i t H v} v \in C\left(\mathbb{R}, H^{1} /\langle x\rangle\right)$ and for some $v_{+} \in\langle x\rangle^{-1} H^{1}$

$$
\left\|v(t)-e^{-i t H} v_{+}\right\|_{H^{1}}=O_{+\infty}\left(t^{-1 / 2}\right),\left\|\langle x\rangle\left(v(t)-e^{-i t H} v_{+}\right)\right\|_{H^{1}} \longrightarrow+\infty 0 .
$$

Moreover, we have $E(\psi)=\left\|\langle\nabla\rangle v_{+}\right\|_{L^{2}}^{2}$, and the correspondence $v(0) \rightarrow v_{+}$defines a bi-Lipschitz map between 0 neighborhoods of $\langle x\rangle^{-1} H^{1}$.

Remark 1 There is some "room" in the above result. Gustafson Nakanishi and Tsai actually solve a kind of quartic NLS for which the critical space is $H^{\frac{5}{6}}$. Moreover, as they mention in [29] the critical weight is rather $\langle x\rangle^{\frac{1}{2}}$. Indeed in this situation we have:

$$
\left\|\epsilon^{i t H} \varphi_{0}\right\|_{L^{3}} \leq \frac{1}{t^{\frac{1}{2}}}\left\|J_{1 / 2} \varphi_{0}\right\|_{L^{2}}, J_{1 / 2}=e^{-i t H}\langle x\rangle^{1 / 2} e^{i t H},
$$

and as mentioned before, a decay of order $t^{-\alpha}, \alpha>-1 / 2$ is required to control of quadratic terms. Scattering below $H^{1}$ would ensure the global existence of strong solutions with infinite energy for (GP). Via the Madelung transform this would give solutions of infinite energy for the Euler Korteweg system (1.1). However, the Proof of Theorem 1.2 relies on some precise explicit computations, whose tractability to fractional Sobolev spaces or weights of fractional power is not clear.

\subsection{Main results}

We recall $V \varphi=\operatorname{Re}(\varphi)+i U \operatorname{Im}(\varphi), U=\sqrt{-\Delta /(2-\Delta)}$. A reminder on Besov spaces is included in Sect. 2.

Theorem 1.3 Let $N \geq 4, u_{0}=\nabla \theta_{0}$. Let $\psi_{0}=\sqrt{\rho_{0}} e^{i \theta_{0}}$ and $\varphi_{0}=\psi_{0}-1, \frac{1}{a^{\prime}}=\frac{1}{2}+\frac{1}{3 N}$. For any $\varepsilon>0$, there exists $\delta>0$ such that if:

$$
\left\|U^{-1} V \varphi_{0}\right\|_{H^{N / 2-\frac{1}{4}+\epsilon} \cap B_{a^{\prime}, 2}^{\frac{N}{2}-\frac{1}{4}+\epsilon}}+\left\|\widehat{\varphi_{0}}\right\|_{L^{1}}<\delta,
$$

then there exists a global weak solution of the system (1.1) satisfying:

$$
\sup _{x, t}|\rho-1| \leq \frac{1}{2}, \quad \rho \in 1+L_{t}^{\infty} H^{\frac{N}{2}-\frac{1}{4}+\epsilon}\left(\mathbb{R}^{N}\right) \text { and } u \in L_{t}^{\infty} H^{\frac{N}{2}-\frac{3}{2}+2 \epsilon}\left(\mathbb{R}^{N}\right) .
$$

If in addition $\varphi_{0} \in H^{\frac{N}{2}+1+\epsilon}$ then the global solution satisfies

$$
\begin{aligned}
(\rho-1, u) \in & \left(L_{l o c}^{\infty} H^{\frac{N}{2}+1+\epsilon}\left(\mathbb{R}^{N}\right) \cap L_{l o c}^{2} B_{\frac{2 N}{N-2}, 2}^{\frac{N}{2}+1+\varepsilon}\right) \\
& \times\left(L_{l o c}^{\infty} H^{\frac{N}{2}+\epsilon}\left(\mathbb{R}^{N}\right) \cap L_{l o c}^{2} B_{\frac{2 N}{N-2}, 2}^{\frac{N}{2}+\epsilon}\left(\mathbb{R}^{N}\right)\right),
\end{aligned}
$$

and is unique in this space.

In dimension $N=3$ the statement is more intricate.

Theorem 1.4 Let $N=3$ and $q_{0}=\rho_{0}-1$ and $u_{0}=\nabla \theta_{0}$. Furthermore $\rho_{0} \in L^{\infty}$ with $\rho_{0} \geq c>0$. Assume that ${ }^{2}\langle x\rangle \nabla \sqrt{\rho_{0}} \in L^{2},\langle x\rangle u_{0} \in L^{2}, q_{0} \in L^{2}, \cos \theta_{0}-1 \in L^{2}$ and

2 These assumptions are the translation of the condition on $\varphi_{0}$ of Theorem 1.2, see (1.14). 
$|x|\left(\sqrt{\rho_{0}} \cos \theta_{0}-1\right) \in L^{2}$. Let $\varphi_{0}=\sqrt{\rho_{0}} e^{i \theta_{0}}-1$, we assume that $\varphi_{0} \in H^{\frac{5}{4}+\epsilon}$ with $\epsilon>0$ and $\left(1+|\xi|^{\epsilon_{1}}\right) \widehat{\varphi_{0}} \in L^{1}$ with $\epsilon_{1}>0$. Then there exists $\delta>0$ depending on $\left\|\varphi_{0}\right\|_{H^{\frac{5}{4}+\epsilon}}$ and $\left\|\left(1+|\xi|^{\epsilon_{1}}\right) \widehat{\varphi_{0}}\right\|_{L^{1}}$ such that if:

$$
\int_{\mathbb{R}^{3}}\left(\langle x\rangle^{2}\left(\left|\nabla \sqrt{\rho_{0}}\right|^{2}+\rho_{0}\left|u_{0}\right|^{2}\right)+\langle x\rangle^{2}\left(\sqrt{\rho_{0}} \cos \left(\theta_{0}\right)-1\right)^{2}\right) d x<\delta,
$$

then there exists a global weak solution $(\rho, u)$ of the system (1.1) such that:

$$
\max \left(\rho, \frac{1}{\rho}\right) \in L^{\infty}\left(\mathbb{R}, L^{\infty}\left(\mathbb{R}^{3}\right)\right), \quad \rho \in 1+L_{\text {loc }}^{\infty}\left(H^{\frac{5}{4}+\epsilon}\left(\mathbb{R}^{3}\right)\right) \text { and } u \in L_{\text {loc }}^{\infty}\left(H^{2 \epsilon}\left(\mathbb{R}^{3}\right)\right) .
$$

If $\varphi_{0} \in H^{\frac{N}{2}+1+\epsilon}$ then the global solution is unique and has the additional regularity:

$$
\rho \in 1+L_{\text {loc }}^{\infty}\left(H^{\frac{N}{2}+1+\epsilon}\left(\mathbb{R}^{3}\right)\right) \text { and } u \in L_{\text {loc }}^{\infty}\left(H^{\frac{N}{2}+\epsilon}\left(\mathbb{R}^{3}\right)\right) \cap L_{\text {loc }}^{2}\left(B_{6,2}^{\frac{N}{2}+\epsilon}\left(\mathbb{R}^{3}\right)\right) .
$$

Remark 2 Let us point out that this result improves the local well-posedness from [5] in two ways: the solutions are global, and at the level of local well-posedness we have a gain of one derivative. This is due to a gain from Strichartz estimates.

Remark 3 Let us mention that global well-posedness remains open in dimension $N=2$ even for small initial data. Scattering is quite unlikely as there exist smooth traveling waves with arbitrarily low energy [7].

Remark 4 We assumed that $\left(1+|\xi|^{\epsilon_{1}}\right) \widehat{\varphi_{0}}$ belongs to $L^{1}$ in order to ensure that $t \rightarrow$ $\left\|e^{-i t H} \varphi_{0}\right\|_{L^{\infty}}$ is continuous in time. To illustrate the importance of this condition, we prove in Theorem 3.1 (following an argument from [9]) that there exists $\varphi_{0}$ arbitrarily small in $L^{\infty} \cap H^{s}$ with $s<\frac{N}{2}$ such that the solution of (GP) blows up in $L^{\infty}$.

Following the same idea than in the previous proofs, we easily get the following results of local existence of strong solution with large initial data. It is an improvement on the regularity required in [5], though in the specific case $\kappa(\rho)=\frac{\kappa_{1}}{\rho}$.

Corollary 1.5 Let $N \geq 3$. Assuming that $\rho_{0} \geq c>0$ and $\varphi_{0}=\left(\sqrt{\rho_{0}} e^{i \theta_{0}}-1\right) \in H^{\frac{N}{2}+1+\epsilon}$ with $u_{0}=\nabla \theta_{0}$ and $\epsilon>0$ then there exist $T>0$ and a local strong solution $(\rho, u)$ on $[0, T)$ of the system (1.1) with the following regularity:

$$
(\rho-1) \in C_{T}\left(H^{\frac{N}{2}+1+\epsilon}\right), u \in C_{T}\left(H^{\frac{N}{2}+\epsilon}\right) \cap L_{T}^{2}\left(\begin{array}{c}
\frac{N}{2}+\epsilon \\
\frac{2 N}{N-2}, 2
\end{array}\right) .
$$

\section{Plan of the paper}

In Sect. 2 we introduce the main technical tools (functional spaces, Strichartz estimates, bilinear product and paraproduct) that will be used. Section 3 is devoted to the Proof of Theorem 1.4, and is split in two parts: first we construct global solutions to (GP) such that $|\psi|$ remains bounded away from 0 . To do this, we control the solution on short time thanks to a Kato smoothing property, while on large time it is sufficient to use the decay from Theorem 1.2. In the subsection for short-time control we also include a general blow up result indicating that there is no hope to get $L^{\infty}$ bounds for initial data that are only in $H^{s}$, $s<N / 2$. Applying the Madelung transform to $\psi$, we construct in Sects. 3.5, resp. 3.6, global weak, resp. strong, solutions. The uniqueness is proved by using the additional integrability 
provided by Strichartz estimates. In Sect. 4.1 we prove a simpler self-contained version of Theorem 1.1 in [27] which is sufficient for the purpose of Sect. 4.2 where we prove Theorem 1.3. In Appendix A we sketch the Proof of Theorem 1.2 from [29] and we highlight the main technical issues.

\section{Main tools}

Throughout the paper, $C$ stands for a constant independent of the parameters in the context. The notation $A \lesssim B$ means that $A \leq C B$. For all Banach space $X$, we denote by $C([0, T], X)$ the set of continuous functions on $[0, T]$ with values in $X$. For $p \in[1,+\infty], L^{p}(0, T, X)$ or $L_{T}^{p}(X)$ is for the set of measurable functions on $(0, T)$ with values in $X$ such that $t \rightarrow\|f(t)\|_{X}$ belongs to $L^{p}(0, T)$.

In this section we recall some notation, definitions and technical tools. We denote the Lebesgue, the Lorentz, the Bessel potential and the Besov spaces as $L^{p}, L^{p, q}, H^{s, p}$ and $B_{p, q}^{s}$ respectively for $1 \leq p, q \leq+\infty$ and $s \in \mathbb{R}$. We denote the Fourier transform on $\mathbb{R}^{N}$ by:

$$
\begin{aligned}
& \mathcal{F} \varphi(\xi)=\widehat{\varphi}(\xi)=\int_{\mathbb{R}^{N}} \varphi(x) e^{-i x \xi} d x, \\
& \mathcal{F}_{x}[f(x, y)](\xi)=\mathcal{F}_{x} f(\xi, y)=\int_{\mathbb{R}^{N}} f(x, y) e^{-i x \xi} d x,
\end{aligned}
$$

and the Fourier multiplier of any function $\varphi$ :

$$
\begin{aligned}
& \varphi(-i \nabla) f=\mathcal{F}^{-1}[\varphi(\xi) \mathcal{F} f(\xi)], \\
& \varphi(-i \nabla)_{x} f(x, y)=\mathcal{F}_{x}^{-1}\left[\varphi(\xi) \mathcal{F}_{x} f(\xi, y)\right] .
\end{aligned}
$$

We follow some notations of [29]. For any number or vector $a$ we denote:

$$
\langle a\rangle=\sqrt{2+|a|^{2}}, \widehat{a}=\frac{a}{|a|}, U(a)=\frac{|a|}{\langle a\rangle}, H(a)=|a|\langle a\rangle .
$$

\subsection{Littlewood-Paley decomposition}

Let $\varphi \in C^{\infty}\left(\mathbb{R}^{N}\right)$ supported in $\left\{\xi \in \mathbb{R}^{N} / \frac{3}{4} \leq|\xi| \leq \frac{8}{3}\right\}, \chi$ supported in the ball $\{\xi \in$ $\left.\mathbb{R}^{N} /|\xi| \leq \frac{4}{3}\right\}$ such that:

$$
\forall \xi \in \mathbb{R}^{N}, \chi(\xi)+\sum_{l \in \mathbb{N}} \varphi\left(2^{-l} \xi\right)=1 \text { if } \xi \neq 0 .
$$

We define the dyadic blocks by:

$$
\begin{array}{lrl}
\Delta_{l} u=0 \text { if } l \leq-2, & \Delta_{-1} u=\chi(D) u=\tilde{h} * u, \\
\Delta_{l} u=\varphi\left(2^{-l} D\right) u \text { if } l \geq 0, & S_{l} u=\sum_{k \leq l-1} \Delta_{k} u .
\end{array}
$$

One can write $u=\sum_{k \in \mathbb{Z}} \Delta_{k} u$ for all temperate distribution. This decomposition is called non-homogeneous Littlewood-Paley decomposition. The homogeneous dyadic blocks are

$$
\dot{\Delta}_{l} u=\varphi\left(2^{-l} D\right) u, \quad l \in \mathbb{Z} .
$$

Note that for $u \in \mathcal{S}^{\prime}$, we have $\sum_{l \in \mathbb{Z}} \dot{\Delta}_{l} u=u$ modulo polynomials. In particular in contrast with the non-homogeneous case we do not have $S_{q} u=\sum_{p \leq q-1} \dot{\Delta}_{p} u$. 
Definition 2.1 Let $1 \leq p, r \leq+\infty$ and $s \in \mathbb{R}$. For $u \in \mathcal{S}^{\prime}\left(\mathbb{R}^{N}\right)$, we set:

$$
\|u\|_{B_{p, q}^{s}}=\left(\sum_{l \in \mathbb{Z}}\left(2^{l s}\left\|\Delta_{l} u\right\|_{L^{p}}\right)^{q}\right)^{\frac{1}{q}} .
$$

The Besov space $B_{p, q}^{s}$ is the set of temperate distribution $u$ such that $\|u\|_{B_{p, q}^{s}}<+\infty$.

Proposition 2.2 The following properties holds:

1. $B_{p, r_{1}}^{s^{\prime}} \hookrightarrow B_{p, r}^{s}$ if $s^{\prime}>s$ or if $s=s^{\prime}$ and $r_{1} \leq r$.

2. $B_{p_{1}, r}^{s} \hookrightarrow B_{p_{2}, r}^{s-N\left(1 / p_{1}-1 / p_{2}\right)}$ for $p_{2} \geq p_{1}$.

3. Real interpolation: if $u \in B_{p, \infty}^{s} \cap B_{p, \infty}^{s^{\prime}}$ and $s<s^{\prime}$ then $u$ belongs to $B_{p, 1}^{\theta s+(1-\theta) s^{\prime}}$ for all $\theta \in(0,1$ and there exists a universal constant $C$ such that:

$$
\|u\|_{B_{p, 1}^{\theta s+(1-\theta) s^{\prime}}} \leq \frac{C}{\theta(1-\theta)\left(s^{\prime}-s\right)}\|u\|_{B_{p, \infty}^{s}}^{\theta}\|u\|_{B_{p, \infty}^{s^{\prime}}}^{1-\theta} .
$$

Let now recall a few product laws in Besov spaces coming directly from the paradifferential calculus of Bony (see $[4,10])$. For $u$ and $v$ two temperate distributions we have the formal decomposition $u v=T_{u} v+T_{v} u+R(u, v)$, with:

$$
\begin{aligned}
& T_{u} v=\sum_{p \leq q-2} \Delta_{p} u \Delta_{q} v=\sum_{q} S_{q-1} u \Delta_{q} v \\
& R(u, v)=\sum_{q} \Delta_{q} u \widetilde{\Delta}_{q} v \text { with } \widetilde{\Delta}_{q}=\Delta_{q-1}+\Delta_{q}+\Delta_{q+1} .
\end{aligned}
$$

Proposition 2.3 Let $p_{1}, p_{2}, r \in[1,+\infty],\left(s_{1}, s_{2}\right) \in \mathbb{R}^{2}$ and $p \in[1,+\infty]$ then we have the following estimates:

- If $\frac{1}{p} \leq \frac{1}{p_{1}}+\frac{1}{p_{2}}$ and $s_{1}+s_{2}+N \inf \left(0,1-\frac{1}{p_{1}}-\frac{1}{p_{2}}\right)>0$ then:

$$
\|R(u, v)\|_{B_{p, r}^{s_{1}+s_{2}}+\frac{N}{p}-\frac{N}{p_{1}}-\frac{N}{p_{2}}} \lesssim\|u\|_{B_{p_{1}, r}^{s_{1}}}\|v\|_{B_{p_{2}, r}^{s_{2}}} .
$$

- If $\frac{1}{p} \leq \frac{1}{p_{1}}+\frac{1}{p_{2}} \leq 1$ and $s_{1}+s_{2}=0$ then:

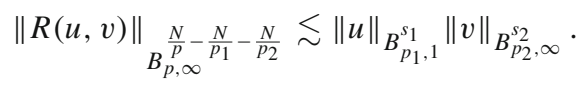

- If $\frac{1}{p} \leq \frac{1}{p_{2}}+\frac{1}{\lambda} \leq 1$ with $\lambda \in[1,+\infty]$ and $p_{1} \leq \lambda$ then:

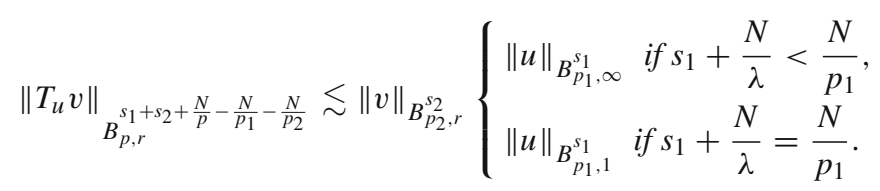

- If $\frac{1}{p} \leq \frac{1}{p_{2}}+\frac{1}{\lambda} \leq 1$ with $\lambda \in[1,+\infty]$ and $p_{1} \leq \lambda$ then:

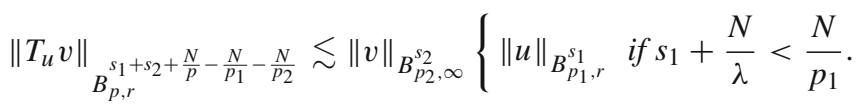


The study of non-stationary PDE's requires space of type $L^{\rho}(0, T, X)$ for appropriate Banach spaces $X$. In our case, we expect $X$ to be a Besov space, so that it is natural to localize the equation through Littlewood-Paley decomposition. But, in doing so, we obtain bounds in spaces which are not type $L^{\rho}(0, T, X)$ (except if $r=p$ ). We are now going to define the spaces of Chemin-Lerner (see [13]) in which we will work, which are a refinement of the spaces $L_{T}^{\rho}\left(B_{p, r}^{s}\right)$.

Definition 2.4 Let $\rho \in[1,+\infty], T \in[1,+\infty]$ and $s_{1} \in \mathbb{R}$. We set:

$$
\|u\|_{\widetilde{L}_{T}^{\rho}\left(B_{p, r}^{s_{1}}\right)}=\left(\sum_{l \in \mathbb{Z}} 2^{l r s_{1}}\left\|\Delta_{l} u(t)\right\|_{L_{T}^{\rho}\left(L^{p}\right)}^{r}\right)^{\frac{1}{r}} .
$$

We then define the space $\widetilde{L}_{T}^{\rho}\left(B_{p, r}^{s_{1}}\right)$ as the set of temperate distribution $u$ over $(0, T) \times \mathbb{R}^{N}$ such that $\|u\|_{\widetilde{L}_{T}^{\rho}\left(B_{p, r}^{s_{1}}\right)}<+\infty$.

We set $\widetilde{C}_{T}\left(\widetilde{B}_{p, r}^{s_{1}}\right)=\widetilde{L}_{T}^{\infty}\left(\widetilde{B}_{p, r}^{s_{1}}\right) \cap C\left([0, T], B_{p, r}^{s_{1}}\right)$. Let us emphasize that, according to Minkowski's inequality, we have:

$$
\|u\|_{\widetilde{L}_{T}^{\rho}\left(B_{p, r}^{s_{1}}\right)} \leq\|u\|_{L_{T}^{\rho}\left(B_{p, r}^{s_{1}}\right)} \text { if } r \geq \rho, \quad\|u\|_{\widetilde{L}_{T}^{\rho}\left(B_{p, r}^{s_{1}}\right)} \geq\|u\|_{L_{T}^{\rho}\left(B_{p, r}^{s_{1}}\right)} \text { if } r \leq \rho .
$$

Remark 5 It is easy to generalize Propositions 2.3 to $\widetilde{L}_{T}^{\rho}\left(B_{p, r}^{s_{1}}\right)$ spaces. The indices $s_{1}, p$, $r$ behave just as in the stationary case whereas the time exponent $\rho$ behaves according to Hölder inequality.

In the sequel we will need a composition lemma in $\widetilde{L}_{T}^{\rho}\left(B_{p, r}^{s}\right)$ spaces (we refer to [4] for a proof).

Proposition 2.5 Let $s>0,(p, r) \in[1,+\infty]$ and $u \in \widetilde{L}_{T}^{\rho}\left(B_{p, r}^{s}\right) \cap L_{T}^{\infty}\left(L^{\infty}\right)$.

1. Let $F \in W_{\text {loc }}^{[s]+2, \infty}\left(\mathbb{R}^{N}\right)$ such that $F(0)=0$. Then $F(u) \in \widetilde{L}_{T}^{\rho}\left(B_{p, r}^{s}\right)$. More precisely there exists a function $C$ depending only on $s, p, r, N$ and $F$ such that:

$$
\|F(u)\|_{\widetilde{L}_{T}^{\rho}\left(B_{p, r}^{s}\right)} \leq C\left(\|u\|_{L_{T}^{\infty}\left(L^{\infty}\right)}\right)\|u\|_{\widetilde{L}_{T}^{\rho}\left(B_{p, r}^{s}\right)} .
$$

2. Let $F \in W_{l o c}^{[s]+3, \infty}\left(\mathbb{R}^{N}\right)$ such that $F(0)=0$. Then $F(u)-F^{\prime}(0) u \in \widetilde{L}_{T}^{\rho}\left(B_{p, r}^{s}\right)$. More precisely there exists a function $C$ depending only on $s, p, r, N$ and $F$ such that:

$$
\left\|F(u)-F^{\prime}(0) u\right\|_{\widetilde{L}_{T}^{\rho}\left(B_{p, r}^{s}\right)} \leq C\left(\|u\|_{L_{T}^{\infty}\left(L^{\infty}\right)}\right)\|u\|_{\widetilde{L}_{T}^{\rho}\left(B_{p, r}^{s}\right)}^{2} .
$$

Let us recall the useful lemma (see [4]).

Lemma 2.6 Let $R_{l}=\left[v, \Delta_{l}\right] \cdot \nabla f$, let $\sigma \in \mathbb{R}$. Assume that $-N \min \left(\frac{1}{p}, \frac{1}{2}\right)<\sigma<\frac{N}{p}+1$ then there exists a constant $C>0$ such that the following inequality is true:

$$
\left\|2^{l \sigma}\right\| R_{l}\left\|_{L^{2}}\right\|_{l^{2}} \leq C\|\nabla v\|_{B_{p, \infty}^{\frac{N}{p}} \cap L^{\infty}}\|f\|_{H^{\sigma}} .
$$




\subsection{Multilinear Fourier multipliers}

For any function $B\left(\xi_{1}, \ldots, \xi_{d}\right)$ on $\left(\mathbb{R}^{N}\right)^{d}$, we associate the $d$-multilinear operator $B\left[f_{1}, \ldots, f_{d}\right]$ defined by:

$$
\mathcal{F}_{x} B\left[f_{1}, \ldots, f_{d}\right]=\int_{\xi=\xi_{1}+\cdots+\xi_{d}} B\left(\xi_{1}, \ldots, \xi_{d}\right) \mathcal{F} f_{1}\left(\xi_{1}\right) \ldots \mathcal{F} f_{d}\left(\xi_{d}\right) d \xi_{2} \ldots d \xi_{d},
$$

which is called a multilinear Fourier multiplier with symbol $B$. We will often identify the symbol to the operator.

Remark 6 For any bilinear symbol $B(\eta, \zeta)$ with $\zeta=\xi-\eta$, we will need to consider derivatives of the symbol with respect to $\eta, \zeta$ or $\xi$. In order to avoid confusions we use the notation

$$
\begin{aligned}
& \left(\nabla_{\xi}^{(\eta)} B, \nabla_{\eta} B\right)=\left(\nabla_{\xi_{2}} B(\eta, \xi-\eta),\left(\nabla_{\xi_{1}}-\nabla_{\xi_{2}}\right) B(\eta, \xi-\eta)\right), \\
& \left(\nabla_{\xi}^{(\zeta)} B, \nabla_{\zeta} B\right)=\left(\nabla_{\xi_{1}} B(\xi-\zeta, \zeta),\left(\nabla_{\xi_{2}}-\nabla_{\xi_{1}}\right) B(\xi-\zeta, \zeta)\right)
\end{aligned}
$$

Under various assumptions, the Hölder type inequality $\left\|B[u, v]_{L^{r}} \lesssim\right\| u\left\|_{L^{p}}\right\| v \|_{L^{q}}, 1 / r=$ $1 / p+1 / q$ is true. The most famous result is due to Coifman and Meyer [14], and will mostly be sufficient for our purpose.

Theorem 2.7 (Coifman-Meyer). Suppose that B satisfies:

$$
\left|\partial_{\xi_{1}}^{\alpha} \partial_{\xi_{2}}^{\beta} B\left(\xi_{1}, \xi_{2}\right)\right| \lesssim \frac{1}{\left(\left|\xi_{1}\right|+\left|\xi_{2}\right|\right)^{|\alpha|+|\beta|}},
$$

for sufficiently many multi-indices $(\alpha, \beta)$. Then the operator:

$$
B[\cdot, \cdot]: L^{p} \times L^{q} \rightarrow L^{r}
$$

is bounded for:

$$
\frac{1}{r}=\frac{1}{p}+\frac{1}{q}, 1<p, q<+\infty \text { and } 1 \leq r<+\infty .
$$

Remark 7 For condition (2.12) to hold, it suffices for $B$ to be homogeneous of degree 0 and of class $C^{\infty}$ on the sphere.

Remark 8 Appendix A includes an interesting result (Lemma A.6) of Gustafson et al on singular bilinear Fourier multipliers, for which there is a loss in the pseudo Hölder inequality: $1 / p+1 / q>1 / r$.

As was shown in [23], one cannot generally replace the right-hand side of (2.12) by $\left|\xi_{1}\right|^{-|\alpha|}\left|\xi_{2}\right|^{-|\beta|}$. Nevertheless, the following estimate from [29] will be useful.

Proposition 2.8 Let $k \in \mathbb{N}$, for any $r, p, q \in(1,+\infty)$ such that $\frac{1}{r}=\frac{1}{p}+\frac{1}{q}$,

$$
\sup _{0 \leq a \leq 1}\left\|\frac{\left\langle\xi_{1}\right\rangle^{2 k(1-a)}\left\langle\xi_{2}\right\rangle^{2 k a}}{\left\langle\left(\xi_{1}, \xi_{2}\right)\right\rangle^{2 k}}[f, g]\right\|_{L^{p_{0}\left(\mathbb{R}^{N}\right)}} \lesssim\|f\|_{L^{p}\left(\mathbb{R}^{N}\right)}\|g\|_{L^{q}\left(\mathbb{R}^{N}\right)} .
$$




\subsection{Strichartz and dispersive estimates}

Lemma 2.9 Let $2 \leq p \leq+\infty, 0 \leq \theta \leq 1, s \in \mathbb{R}$, and $\sigma=\frac{1}{2}-\frac{1}{p}$. Then we have:

$$
\left\|e^{-i t H} v\right\|_{B_{p, 2}^{s}} \lesssim|t|^{-(N-\theta) \sigma}\left\|U^{(N-2+3 \theta) \sigma}\langle\nabla\rangle^{2 \theta \sigma} v\right\|_{B_{p^{\prime}, 2}^{s}},
$$

where $p^{\prime}=\frac{p}{p-1}$ is the Hölder conjugate. For $2 \leq p<+\infty$, we have also:

$$
\left\|e^{-i t H} v\right\|_{L^{p, 2}} \lesssim|t|^{-(N-\theta) \sigma}\left\|U^{(N-2+3 \theta) \sigma}\langle\nabla\rangle^{2 \theta \sigma} v\right\|_{L^{p^{\prime}, 2}} .
$$

Let us recall the Strichartz estimate for the operator $H$, we recall here a proposition due to Gustafson et al. in [27,28].

Proposition 2.10 For $j=1,2$, let $2 \leq p_{j}, q_{j} \leq+\infty, \frac{2}{q_{j}}+\frac{N}{p_{j}}=\frac{N}{2}$ and $s_{j}=\frac{N-2}{2}\left(\frac{1}{2}-\frac{1}{p_{j}}\right)$ but $\left(q_{j}, p_{j}\right) \neq(2,+\infty)$. Then we have:

$$
\begin{aligned}
& \left\|e^{-i H t} \Delta_{j} \varphi\right\|_{L^{q_{1}}\left(L^{p_{1}}\right)} \leq\left\|U^{s_{1}} \Delta_{j} \varphi\right\|_{L^{2}}, \\
& \left\|e^{-i H t} \varphi\right\|_{L^{q_{1}}\left(B_{p_{1}, 2}^{s}\right)} \lesssim\left\|U^{s_{1}} \varphi\right\|_{B_{2,2}^{s}}, \\
& \left\|\int_{0}^{t} e^{-i(t-s) H} \Delta_{j} f\right\|_{L^{q_{1}}\left(L^{p_{1}}\right)} \leq\left\|U^{s_{1}+s_{2}} \Delta_{j} f\right\|_{L^{q_{2}^{\prime}}\left(L^{p_{2}^{\prime}}\right)}, \\
& \left\|\int_{0}^{t} e^{-i(t-s) H} f\right\|_{L^{q_{1}}\left(B_{p_{1}, 2}^{s}\right)} \lesssim\left\|U^{s_{1}+s_{2}} f\right\|_{L^{q_{2}^{\prime}}\left(B_{p_{2}^{\prime}, 2}^{s}\right)} .
\end{aligned}
$$

Remark 9 These Strichartz estimates are very close to the classical one for Schrödinger equations except in low frequencies.

\section{Proof of Theorem 1.4}

In order to prove Theorem 1.4, it is enough to prove that the global strong solution $\psi$ of (GP) obtained by Gustafson et al. in [27] does not vanish, which is implied by the smallness of $\|\varphi\|_{L_{x, t}^{\infty}}$. This is the aim of Sects. 3.1 to 3.3, then via the Madelung transform we propagate the regularity of $\varphi$ on $\rho-1$ and $u=\nabla \theta$. It is enough to obtain the existence of global solutions to the system (1.1), uniqueness is then derived with an energy argument in Sect. 3.6.

\section{Smallness of $\|\varphi\|_{L^{\infty}}$ norm}

The Gross-Pitaevskii equation is known to be globally well-posed in the energy space $\{u \in$ $\left.H_{l o c}^{1}\left(\mathbb{R}^{3}\right): \nabla u \in L^{2},|u|^{2}-1 \in L^{2}\left(\mathbb{R}^{3}\right)\right\}$ (see [18]). Moreover, it propagates regularity: for any $s>0$ if $\varphi_{0} \in H^{s}\left(\mathbb{R}^{3}\right)$ then the solution $\varphi$ is bounded in $L_{\text {loc }}^{\infty}\left(H^{s}\left(\mathbb{R}^{3}\right)\right)$. However, from [18] we cannot deduce that this norms remains small uniformly in time, and therefore we can not estimate the $L^{\infty}$ norm of $\frac{1}{|\psi|^{2}}$ (which is necessary in order to use the Madelung transform as it corresponds to $\frac{1}{\rho}$ ).

Furthermore, the $L^{\infty}$ regularity is not propagated by the semi-group $e^{i t \Delta}$ which explains why we need to use stronger spaces than the naive choice $L^{\infty} \cap H^{1}$. However, in order to exploit the time decay we will split the analysis between short and long time. In long time using weighted data (see Theorem 1.2 of Gustafson et al.) ensures a small $L^{\infty}$ control of $\varphi$. In short time we will use a smoothing effect on the Duhamel part in order to control the $L^{\infty}$ norm with initial data in $H^{N / 2-1 / 6+\epsilon}$ rather than $H^{\frac{N}{2}+\epsilon}$ with $\epsilon>0$. 


\section{1 $L^{\infty}$ control of $\varphi$ in large time $t \geq \alpha>0$}

According to (A.11) in Appendix A:

$$
\begin{aligned}
& \left\||\nabla|^{-2+\frac{5 \theta}{3}} v_{<1}(t)\right\|_{L^{6}} \lesssim \min \left(1, t^{-\theta}\right)\|v(t)\|_{X(t)}, \\
& \left\||\nabla|^{\theta} v_{\geq 1}(t)\right\|_{L^{6}} \lesssim \min \left(t^{-\theta}, t^{-1}\right)\|v(t)\|_{X(t)},
\end{aligned}
$$

with $0 \leq \theta \leq 1$. In particular it implies that:

$$
\left\|U^{-1} v_{<1}(t)\right\|_{L^{6}}+\left\|\nabla U^{-1} v_{\geq 1}(t)\right\|_{L^{6}} \lesssim\left(\min \left(1, t^{-\frac{3}{5}}\right)+\frac{1}{t}\right)\|v(t)\|_{X(t)} .
$$

Since $\varphi=V^{-1} v=\operatorname{Re} v+i U^{-1} \operatorname{Im} v$ we have for $t \geq \alpha$ (which will be fixed in Sect. 3.3) and by Sobolev embedding:

$$
\begin{aligned}
&\|\varphi\|_{L_{t \geq \alpha}^{\infty}\left(H^{1,6}\right)} \lesssim\left\|V^{-1} v\right\|_{L_{t \geq 1}^{\infty}\left(H^{1,6}\right)} \lesssim \frac{1}{t^{\frac{3}{5}}}\|v\|_{X(t)}, \\
&\|\varphi\|_{L_{t \geq \alpha}^{\infty}\left(L^{\infty}\right)} \lesssim \frac{1}{t^{\frac{3}{5}}}\|v\|_{X(t)} \lesssim \frac{\delta}{t^{\frac{3}{5}}} \leq \frac{\delta}{\alpha^{3 / 5}} .
\end{aligned}
$$

\subsection{A smoothing property}

It remains now to show that $\psi$ does not cancel on the time interval $[0, \alpha]$. To do this we show that $\varphi$ is small in $L^{\infty}$ by using a Kato smoothing property which gives us a gain of half a derivative for the integral part in the Duhamel formula. This is a relatively well-known property that seems to have been explicitly stated only recently by Bona et al. in [9]. This was used to prove a dispersive blow up result for Schrödinger and Gross-Pitaevskii equations. We include their result in this section as it enlightens the fact that $L^{\infty}$ is a "bad" space for initial data.

Theorem 3.1 (Bona et al. [9])

For $N \geq 3, s>\frac{N}{2}-\frac{1}{4}, \varphi_{0} \in H^{s}\left(\mathbb{R}^{N}\right)$, let $\varphi$ be the solution of (1.8) satisfying:

$$
\begin{aligned}
\left(\begin{array}{c}
\operatorname{Re}(\varphi) \\
\operatorname{Im}(\varphi)
\end{array}\right)(t) & =A(t)\left(\begin{array}{c}
\operatorname{Re}\left(\varphi_{0}\right) \\
\operatorname{Im}\left(\varphi_{0}\right)
\end{array}\right)+\int_{0}^{t} A(t-s)\left(\begin{array}{c}
\operatorname{Re}(F(\varphi(s)) \\
\operatorname{Im}(F(\varphi(s))
\end{array}\right) d s \\
& =A(t)\left(\begin{array}{l}
\operatorname{Re}\left(\varphi_{0}\right) \\
\operatorname{Im}\left(\varphi_{0}\right)
\end{array}\right)+I(t, x) .
\end{aligned}
$$

with

$$
A(t)=\left(\begin{array}{cc}
\cos (H t) & U \sin (H t) \\
-U^{-1} \sin (H t) & \cos (H t)
\end{array}\right) .
$$

Let $\varphi_{0} \in H^{s}$ then there exists $0<T<1$ such that (3.4) has a solution $\varphi$ on $[0, T]$ which verifies $\varphi \in S_{T}$ with $S_{T}=C\left([0, T], H^{s}\right) \cap L^{2}\left([0, T], H^{s, \frac{2 N}{N-2}}\right)$. Furthermore we have:

1. there exists $\beta_{1}>0$ such that

$$
\begin{aligned}
\varphi(t) & =e^{i t(\Delta-1)} \varphi_{0}+\int_{0}^{t} e^{i(t-s)(\Delta-1)} F(\varphi) d s+g(t),\|g\|_{L_{T}^{\infty} H^{s+1}} \\
& \lesssim T^{\beta_{1}}\left(\left\|\varphi_{0}\right\|_{H^{s}}+\left\|\varphi_{0}\right\|_{H^{s}}^{3}\right) .
\end{aligned}
$$


2. $\int_{0}^{t} e^{i(t-s)(\Delta-1)} F(\varphi) d s \in C\left([0, T], H^{s+\frac{1}{2}}\right)$, in particular $I \in C_{b}\left([0, T] \times \mathbb{R}^{N}\right)$ and there exists $\beta>0$ :

$$
\|I\|_{C_{b}\left([0, T] \times \mathbb{R}^{N}\right)} \lesssim T^{\beta}\left(\left\|\varphi_{0}\right\|_{H^{s}}^{2}+\left\|\varphi_{0}\right\|_{H^{s}}^{3}\right) .
$$

3. Moreover, for $s<N / 2, T>0$, there exists $\varepsilon_{0}>0$, such that for any $\varepsilon \leq \varepsilon_{0}$ there exists $\varphi_{0} \in H^{s}\left(\mathbb{R}^{3}\right) \cap C^{\infty}\left(\mathbb{R}^{3}\right)$ with $\left\|\varphi_{0}\right\|_{H^{s} \cap L^{\infty}} \leq \varepsilon$, the solution is in $S_{T}$ and $\|\varphi(\cdot, T)\|_{L^{\infty}\left(\mathbb{R}^{3}\right)}=+\infty$.

Remark 10 - This is essentially is a linear result. The blow up of the $L^{\infty}$ norm is due to the linear evolution part while nonlinear terms remain bounded. It can be proved (using invariances of the equations) that for any $\left(x_{0}, t_{0}\right) \in \mathbb{R}^{d} \times \mathbb{R}^{*}$ there exists an initial data such that the solution blows up in $L^{\infty}$ precisely at $\left(x_{0}, t_{0}\right)$.

- Actually the results from [9] are slightly different from the statement of Theorem 3.1. For the comfort of the reader we include a short proof.

Proof We first prove (1). Consider the Cauchy problem

$$
\left\{\begin{array}{l}
i \partial_{t} \varphi+\Delta \varphi-2 \operatorname{Re}(\varphi)=(\varphi+1)|\varphi|^{2}+2 \operatorname{Re}(\varphi) \varphi=F(\varphi), \\
\left.\varphi\right|_{t=0}=\varphi_{0}
\end{array}\right.
$$

Since $s>N / 2-1$ (the critical index for cubic NLS) the existence of a solution in $S_{T}:=$ $C\left([0, T], H^{s}\right) \cap L^{2}\left([0, T], H^{s, \frac{2 N}{N-2}}\right)$ follows from the standard theory (it suffices to consider $\operatorname{Re} \varphi$ as a source term, see [12]). Taking $\varphi_{0}$ in $H^{s}$ ensures the existence of a strong solution on an interval $[0, T]$ ( $T$ depends on $\left\|\varphi_{0}\right\|_{H^{s}}$ since we are subcritical and in addition we assume that $T<1$ ) with in addition: $\|\varphi\|_{S_{T}} \lesssim\left\|\varphi_{0}\right\|_{H^{s}}$.

We remind the notations:

$$
H=\sqrt{-\Delta(2-\Delta)}, U=\sqrt{\frac{-\Delta}{2-\Delta}}, \text { and } A(t)=\left(\begin{array}{cc}
\cos (H t) & U \sin (H t) \\
-U^{-1} \sin (H t) & \cos (H t)
\end{array}\right),
$$

the Duhamel formula reads

$$
\left(\begin{array}{l}
\operatorname{Re}(\varphi) \\
\operatorname{Im}(\varphi)
\end{array}\right)(t)=A(t)\left(\begin{array}{l}
\operatorname{Re}\left(\varphi_{0}\right) \\
\operatorname{Im}\left(\varphi_{0}\right)
\end{array}\right)+\int_{0}^{t} A(t-s)\left(\begin{array}{l}
\operatorname{Re}(F(\varphi(s)) \\
\operatorname{Im}(F(\varphi(s))
\end{array}\right) d s
$$

The linear evolution operator $A(t)$ can be compared with the Schrödinger evolution group $A_{S}=\left(\begin{array}{cc}\cos (-\Delta t) & \sin (-\Delta t) \\ -\sin (-\Delta t) & \cos (-\Delta t)\end{array}\right)$ by Taylor expansion : there exists $C_{1}$ such that

$$
\left.\left(1+|\xi|^{2}\right)|H(\xi)-| \xi\right|^{2}-1|+| U(\xi)-1\left|\leq C_{1}, \quad\left(|\xi|+|\xi|^{2}\right)\right| U^{-1}(\xi)-1 \mid \leq C_{1} .
$$

We can deduce directly from the mean value theorem that there exists $C>0$ such that:

$$
\left(1+|\xi|^{2}\right)\|R(\xi, t)\|:=\left(1+|\xi|^{2}\right)\left\|A(\xi, t)-\left(\begin{array}{cc}
\cos \left(\left(|\xi|^{2}+1\right) t\right) & \sin \left(\left(|\xi|^{2}+1\right) t\right) \\
\left.-\sin \left(|\xi|^{2}+1\right) t\right) & \cos \left(\left(|\xi|^{2}+1\right) t\right)
\end{array}\right)\right\| \leq C t
$$

(the singularity of $U^{-1}$ in low frequencies is harmless since there is a factor $\sin (H t)$ which cancels at the same order). The associated operator $R(\Delta, t)$ is thus continuous $H^{s} \rightarrow H^{s+2}$, and setting $M(t)=\left(\begin{array}{cc}\cos t & \sin t \\ -\sin t & \cos t\end{array}\right)$ the solution rewrites for any $\left.\left.t \in\right] 0, T\right]$ :

$$
\begin{aligned}
\left(\begin{array}{c}
\operatorname{Re}(\varphi) \\
\operatorname{Im}(\varphi)
\end{array}\right)(t)= & A_{S}(t) M(t)\left(\begin{array}{c}
\operatorname{Re}\left(\varphi_{0}\right) \\
\operatorname{Im}\left(\varphi_{0}\right)
\end{array}\right)+\int_{0}^{t} A_{S}(t-s) M(t-s)\left(\begin{array}{l}
\operatorname{Re}(F(\varphi(s)) \\
\operatorname{Im}(F(\varphi(s))
\end{array}\right) d s \\
& +R(t)\left(\begin{array}{c}
\operatorname{Re}\left(\varphi_{0}\right) \\
\operatorname{Im}\left(\varphi_{0}\right)
\end{array}\right)+\int_{0}^{t} R(t-s)\left(\begin{array}{c}
\operatorname{Re}(F(\varphi(s)) \\
\operatorname{Im}(F(\varphi(s))
\end{array}\right) d s,
\end{aligned}
$$


with

$$
\left\|R(t)\left(\begin{array}{l}
\operatorname{Re}\left(\varphi_{0}\right) \\
\operatorname{Im}\left(\varphi_{0}\right)
\end{array}\right)\right\|_{H^{s+2}} \lesssim t\left\|\varphi_{0}\right\|_{H^{s}}
$$

For the nonlinear term of the second line we have $\varphi \in C_{T} H^{s} \cap L_{T}^{p} H^{s, q}$, for any admissible $(p, q)$. Taking $q=\frac{N}{s}+\epsilon$ with $\epsilon>0$ sufficiently small and $s<\frac{N}{2}$ such that $2<q<\frac{2 N}{N-2}$, $p>2$ and $H^{s, q} \hookrightarrow L^{\infty}$. Using product rules in Sobolev spaces, we have:

$$
\begin{aligned}
\left\|\varphi^{2}\right\|_{H^{s-1}} & \lesssim\|\varphi\|_{H^{s}}\|\varphi\|_{L^{\infty}} \lesssim\|\varphi\|_{H^{s}}\|\varphi\|_{H^{s, q}}, \\
\left\|\varphi^{3}\right\|_{H^{s-1}} & \lesssim\|\varphi\|_{H^{s}}\|\varphi\|_{H^{s, q}}^{2},
\end{aligned}
$$

so that putting these estimates in $\int_{0}^{t} R(t-s) F(\varphi) d s$

$$
\begin{aligned}
\left\|\int_{0}^{t} R(t-s)\left(\begin{array}{l}
\operatorname{Re}(F(\varphi(s)) \\
\operatorname{Im}(F(\varphi(s))
\end{array}\right) d s\right\|_{H^{s+1}} & \lesssim\|F(\varphi)\|_{L_{T}^{1} H^{s-1}} \\
& \lesssim T^{\frac{p-2}{p}}\|\varphi\|_{L_{T}^{\infty} H^{s}}\left(\|\varphi\|_{L_{T}^{p} H^{s, q}}+\|\varphi\|_{L_{T}^{p} H^{s, q}}^{2}\right) \\
& \lesssim T^{\frac{p-2}{p}}\left(\|\varphi\|_{S_{t}}^{2}+\|\varphi\|_{S_{T}}^{3}\right) .
\end{aligned}
$$

Indeed we recall that the norm $S_{T}$ enables to control all the Strichartz norm. The estimate $\|\varphi\|_{S_{T}} \lesssim\left\|\varphi_{0}\right\|_{H^{s}}$ ends the proof of 1).

The first line of (3.8) rewrites in complex coordinates as $e^{i t(\Delta-1)} \varphi_{0}+\int_{0}^{t} e^{i(t-s)(\Delta-1)}$ $F(\varphi(s)) d s$, so that points (2) and (3) are precisely ${ }^{3}$ Proposition 4.1 and Lemma 2.1 from [9].

For completeness we mention the argument for point 3): the function $\varphi_{0}=\frac{e^{-i x^{2} / 4}}{\left(1+x^{2}\right)^{m / 2}}$ belongs to $H^{s}$ for $m>s+N / 2$ (and obviously $C^{\infty}\left(\mathbb{R}^{3}\right) \cap L^{\infty}$ ) and the linear solution to the corresponding Cauchy problem $e^{i t \Delta} \varphi_{0}$ blows up precisely at time $t_{b}=1$, and at the point $x_{b}=0$ if $m \leq 3$. This follows from the explicit formula

$$
e^{i t \Delta} \varphi_{0}(x)=\frac{1}{(4 i \pi t)^{3 / 2}} \int_{\mathbb{R}^{3}} e^{i \frac{|x-y|^{2}}{4 t}} \varphi_{0}(y) d y=\frac{1}{(4 i \pi t)^{3 / 2}} \int_{\mathbb{R}^{3}} e^{i \frac{|x-y|^{2}}{4 t}} d y \frac{e^{-i y^{2} / 4}}{\left(1+y^{2}\right)^{m / 2}} d y,
$$

which holds for all $(x, t) \neq(0,1)$ (it can be rigorously justified by oscillating integrals arguments). For $(x, t)=(0,1), 1 /\left(1+|x|^{2}\right)^{m / 2}$ is not integrable iff $m \leq N$ which gives $L^{\infty}$ blow up. Blow up for $T \neq 1$ and small data is easily obtained by a scaling argument.

\subsection{Global $L^{\infty}$ control of $\varphi$}

We recall the assumption of Theorem 1.4: $\left(1+|\xi|^{\varepsilon_{1}}\right) \widehat{\varphi_{0}} \in L^{1}$. Let us consider $e^{i t(\Delta-1)} \varphi_{0}$ in (3.6) and set

$$
C(t, x)=\varphi_{0}(x)-e^{i t(\Delta-1)} \varphi_{0}(x)=\frac{1}{(2 \pi)^{N}} \int_{\mathbb{R}^{3}} e^{i x \cdot \xi}\left(1-e^{-i t\left(1+|\xi|^{2}\right)}\right) \widehat{\varphi_{0}}(\xi) d \xi .
$$

3 The smoothing property of from [9] is actually stated for nonlinearities of type $|u|^{\alpha} u$ but can be carried out without any change to our case. There is also a condition $[\alpha+1] \geq s+1 / 2$ however it is only required to differentiate the function $u \rightarrow|u|^{p} u$, in our case the nonlinearity is polynomial so this is not an issue. 
To estimate this term we fix $M>0$, we have for all $(t, x) \in[0, T] \times \mathbb{R}^{3}$ :

$$
\left|\int_{\mathbb{R}^{3}} e^{i x \cdot \xi}\left(1-e^{-i t\left(1+|\xi|^{2}\right)}\right) \widehat{\varphi_{0}}(\xi) d \xi\right| \leq t\left(1+M^{2}\right)\left\|\widehat{\varphi_{0}}\right\|_{L^{1}}+\frac{2}{M^{\epsilon_{1}}}\left\|\xi^{\epsilon_{1}} \widehat{\varphi_{0}}\right\|_{L^{1}} .
$$

Let us mention in addition that $e^{i t(\Delta-1)} \varphi_{0}$ belongs to $L^{\infty}\left(\mathbb{R}^{+}, L^{\infty}\right)$. Let us look now at the density $\rho$ when $t \in[0, T]$ :

$$
\begin{aligned}
& \rho(t, \cdot)=|\psi(t, \cdot)|^{2}=|1+\varphi(t, \cdot)|^{2} \\
&=\left(1+\operatorname{Re}\left(e^{i t(\Delta-1)} \varphi_{0}\right)+\operatorname{Re}(I(t)+g(t))\right)^{2}+\left(\operatorname{Im}\left(e^{i t(\Delta-1)} \varphi_{0}\right)+\operatorname{Im}(I(t)+g(t))\right)^{2} \\
& \geq\left(1+\operatorname{Re}\left(e^{i t(\Delta-1)} \varphi_{0}\right)\right)^{2}-|I(t)+g(t)|^{2}-2\left|1+\operatorname{Re}\left(e^{i t(\Delta-1)} \varphi_{0}\right)\right||I(t)+g(t)| \\
&+\left(\operatorname{Im}\left(e^{i t(\Delta-1)} \varphi_{0}\right)\right)^{2}-|I(t)+g(t)|^{2}-2\left|\operatorname{Im}\left(e^{i t(\Delta-1)} \varphi_{0}\right)\right||I(t)+g(t)| \\
& \geq \rho_{0}(x)-|I(t)+g(t)|^{2}-2\left|1+\operatorname{Re}\left(e^{i t(\Delta-1)} \varphi_{0}\right)\right||I(t)+g(t)| \\
&-|I(t)+g(t)|^{2}-2\left|\operatorname{Im}\left(e^{i t(\Delta-1)} \varphi_{0}\right)\right||I(t)+g(t)|-2 \operatorname{Re} C\left(1+\operatorname{Re}\left(\varphi_{0}\right)\right)-(\operatorname{Re} C)^{2} \\
&-2 \operatorname{Im} C \operatorname{Im}\left(\varphi_{0}\right)-(\operatorname{Im} C)^{2} .
\end{aligned}
$$

We deduce from (3.9), (3.6), (3.7) and using the assumption $\rho_{0}=\left|1+\varphi_{0}\right|^{2} \geq c$ that there exists $t_{1}$ sufficiently small and $c^{\prime}>0$ such that:

$$
\rho(s, x) \geq c^{\prime} \text { for all }(s, x) \in\left[0, t_{1}\right] \times \mathbb{R}^{N} .
$$

We fix now the $\alpha$ of Sect. 3.1 such that $t_{1}=\alpha$. Combining now (3.3) and (3.10), and taking $\delta$ small enough ( $\delta$ depends on $\varphi_{0}$ ) we obtain:

$$
|\psi(t, x)|^{2}=\rho(t, x) \geq \frac{c^{\prime}}{2} \forall t \geq 0 \text { and } \forall x \in \mathbb{R}^{3} .
$$

Remark 11 It will sufficient in the following to proves that the solution $(\rho, u)$ of system (1.1) has no vacuum for $t \geq 0$.

\subsection{How to propagate the regularity from $\varphi$ to $\rho$ and $u$}

Theorem 1.2 ensures the existence of a unique global solution $\varphi$ to the system (1.8) with in addition $\varphi \in C\left(\mathbb{R}^{+}, H^{1}\left(\mathbb{R}^{3}\right)\right) \cap L^{2}\left(\mathbb{R}^{+}, H^{1,6}\right)$. Indeed (see Appendix (A.12)) $U^{-1} v \in$ $L^{2}\left(H^{1,6}\right)$. In this section, we use the Madelung transform and composition results in Sobolev spaces to estimate $\rho$ and $u$. Up to a change of variables we can assume $\kappa_{1}=1$, which we will do from now on (see however our remarks in the Appendix B on how the smallness condition depends of $\kappa_{1}$ ).

Remark 12 In this section in order to prove the existence of global strong solution for the system (1.1) we propagate high regularity of $\varphi$ on $\rho$ and $u$. Then according to standard theory of nonlinear Schrödinger equations $\varphi$ belongs to $C\left((0, T), H^{\frac{N}{2}+1+\epsilon}\left(\mathbb{R}^{3}\right)\right)$ for any $T>0$ (see [12], Theorems 5.3.1, p. 146 and 5.4.1, p. 146) for any $T>0$. Moreover, using Strichartz estimates one can check also $\varphi \in L_{T}^{2}\left(B_{6,2}^{\frac{N}{2}+1+\epsilon}\right)$.

Proposition 3.2 Let $N=3$. Assume that the solution $\varphi$ of $(1.8)$ belongs to $C([0,+\infty)$, $\left.H^{s}\left(\mathbb{R}^{3}\right)\right) \cap L^{2}\left([0, T], B_{6,2}^{s}\right)$ for any $T>0$ with $s>\frac{3}{2}$ and that $|\psi(t, x)|=|1+\varphi(t, x)| \geq c_{1}$ 
for all $(t, x) \in \mathbb{R}^{+} \times \mathbb{R}^{3}$ then we have:

$$
q=\rho-1 \in L_{T}^{\infty}\left(H^{s}\left(\mathbb{R}^{3}\right)\right) \cap L_{T}^{2}\left(B_{6,2}^{s}\right) \text { and } u \in L^{\infty}\left(H^{s-1}\left(\mathbb{R}^{3}\right)\right) \cap L_{T}^{2}\left(B_{6,2}^{s-1}\right) .
$$

More precisely we have for any $T>0$ :

$$
\begin{aligned}
& \|q\|_{L_{T}^{\infty}\left(H^{s}\right) \cap L_{T}^{2}\left(B_{6,2}^{s}\right)} \lesssim\left(1+\|\varphi\|_{L_{T, x}^{\infty}}\right)\|\varphi\|_{L_{T}^{\infty}\left(H^{s}\right) \cap L_{T}^{2}\left(B_{6,2}^{s}\right)}, \\
& \|u\|_{L_{T}^{\infty}\left(H^{s-1}\right)} \lesssim\left(1+C_{1}\left(\|\varphi\|_{\left.L_{T, x}^{\infty}\right)}\right)\left(1+\|\varphi\|_{L_{T}^{\infty}\left(H^{s}\right)}\right)\right)\|\varphi\|_{L_{T}^{\infty}\left(H^{s}\right)}, \\
& \|u\|_{L_{T}^{2}\left(B_{6,2}^{s-1}\right)} \lesssim\left(1+C_{2}\left(\|\varphi\|_{L_{T, x}^{\infty}}\right)\left(1+\|\varphi\|_{L_{T}^{\infty}\left(H^{s}\right)}\right)\right)\|\varphi\|_{L_{T}^{2}\left(B_{6,2}^{s}\right)} .
\end{aligned}
$$

Remark 13 A similar result holds when $N \geq 4$ and the solution of (1.8) belongs to

$$
C\left([0,+\infty), H^{s}\left(\mathbb{R}^{N}\right)\right) \cap L^{2}\left([0, T], B_{\frac{2 N}{N-2}, 2}^{s}\right) \text { for any } T>0 \text { with } s>\frac{N}{2} .
$$

Proof We are now interested in translating the regularity of $\varphi$ on $\rho$ and $u$ via the Madelung transform:

$$
\psi(t, x)=1+\varphi(t, x)=\sqrt{\rho}(t, x) e^{i \theta(t, x) / 2} \text { with } u=\nabla \theta
$$

In particular we are going to use a polar decomposition (used also in [2]):

$$
\begin{aligned}
& \tau(t, x)=\frac{\psi(t, x)}{|\psi(t, x)|}=\left(\frac{1+\varphi(t, x)}{|1+\varphi(t, x)|}-1\right)+1, \\
& q(t, x)=2 \operatorname{Re}(\varphi)+|\varphi|^{2}, \\
& u(t, x)=\nabla \theta(t, x)=\operatorname{Im}\left(\left[\left(\frac{1+\overline{\varphi(t, x)}}{|1+\varphi(t, x)|^{2}}-1\right) \nabla \varphi(t, x)+\nabla \varphi(t, x)\right]\right) .
\end{aligned}
$$

Let us point out that $u$ is well defined on $(0,+\infty) \times \mathbb{R}^{N}$ since we have assumed that $|\psi| \geq c_{1}$. We have now by Propositions (2.3) and (2.5) (since $2 s-1-\frac{N}{2} \geq s-1$ ):

$$
\begin{aligned}
\|q\|_{L_{T}^{\infty}\left(H^{s}\right) \cap L_{T}^{2}\left(B_{6,2}^{s}\right)} & \lesssim\left(1+\|\varphi\|_{L_{T, x}^{\infty}}\right)\|\varphi\|_{L_{T}^{\infty}\left(H^{s}\right) \cap L_{T}^{2}\left(B_{6,2}^{s}\right)}, \\
\|u\|_{L_{T}^{\infty}\left(H^{s-1}\right)} \lesssim\left(1+\left\|\left(\frac{1+\bar{\varphi}(t, x)}{|1+\varphi(t, x)|^{2}}-1\right)\right\|_{L_{T, x}^{\infty}}\right)\|\nabla \varphi\|_{L_{T}^{\infty}\left(H^{s-1}\right)} & +\left\|\left(\frac{1+\bar{\varphi}(t, x)}{|1+\varphi(t, x)|^{2}}-1\right)\right\|_{L_{T}^{\infty}\left(H^{s}\right)}\|\nabla \varphi\|_{L_{T}^{\infty}\left(H^{s-1}\right)} \\
& \quad\left(1+C_{1}\left(\|\varphi\|_{L_{T, x}^{\infty}},\left\|\frac{1}{|\psi|}\right\|_{\left.L_{T, x}^{\infty}\right)}\left(1+\|\varphi\|_{L_{T}^{\infty}\left(H^{s}\right)}\right)\right)\|\varphi\|_{L_{T}^{\infty}\left(H^{s}\right)} .\right.
\end{aligned}
$$

In the same way we have:

$$
\|u\|_{L_{T}^{2}\left(B_{6,2}^{s-1}\right)} \lesssim\left(1+C_{2}\left(\|\varphi\|_{L_{T, x}^{\infty}},\left\|\frac{1}{|\psi|}\right\|_{L_{T, x}^{\infty}}\right)\left(1+\|\varphi\|_{L_{T}^{\infty}\left(H^{s}\right)}\right)\right)\|\varphi\|_{L_{T}^{2}\left(B_{6,2}^{s}\right)} .
$$




\subsection{Existence of global weak solution when $N=3$}

\section{Smooth initial data}

We first assume that the initial data $\left(\rho_{0}, u_{0}\right)$ are smooth: in this case $\varphi_{0}$ is in $H^{s}\left(\mathbb{R}^{3}\right)$ with $s$ large. Using propagation of the regularity (see Cazenave [12], Chapter 5) the solution $\varphi(t)$ constructed in Theorem 1.2 is in $C\left([0, T], H^{s}\left(\mathbb{R}^{3}\right)\right)$ for any $T>0$. In particular taking $s$ large enough, by Sobolev embedding $\varphi$ belongs to $C^{3}\left(\mathbb{R} \times \mathbb{R}^{3}\right)$. It implies that $\varphi$ is a classical solution of Gross-Pitaevskii equation and we are going to exhibit a solution $(\rho, u)$ of the system (1.1) using the Madelung transform. Setting:

$$
\rho=|1+\varphi|^{2} \text { and } u=2 \operatorname{Im}\left(\nabla \varphi \frac{1+\bar{\varphi}}{|1+\varphi|^{2}}\right),
$$

it is clear that $u$ is well defined since $|1+\varphi| \geq \sqrt{\frac{c^{\prime}}{2}}$ (see Sect. 3.3). In addition $(\rho, u)$ is in $C^{3}\left(\mathbb{R} \times \mathbb{R}^{3}\right) \times C^{2}\left(\mathbb{R} \times \mathbb{R}^{3}\right)$ so that the formal Madelung transform leading from (GP) to (EK) is actually rigorously defined and $(\rho, u)$ is a global classical solution of the system (1.1).

\section{General case}

Let us now treat the general case where $\left(\rho_{0}, u_{0}\right)$ verify the assumption of Theorem 1.4 with $\varphi_{0} \in H^{\frac{5}{4}+\epsilon}\left(\mathbb{R}^{3}\right)$ in particular. We set $\varphi_{0}^{n}=\varphi_{0} * \psi_{n}$ with $\psi_{n}=n^{3} \psi(n \cdot)$ a regularizing kernel (with $\psi \in C_{0}^{\infty}\left(\mathbb{R}^{3}\right), \int \psi(y) d y=1,0 \leq \psi \leq 1$ and $\operatorname{supp} \psi_{n} \subset B(0,1)$ ) such that $\varphi_{0}^{n}$ belongs to $H^{s}$ for any $s$ large enough. We are interesting in showing that $\varphi_{0}^{n}$ verify the assumptions of Theorem 1.2. Assume that $\langle x\rangle f \in L^{2}\left(\mathbb{R}^{3}\right)$ then we have by Hölder's inequality and Fubini theorem:

$$
\begin{aligned}
& \int\langle x\rangle^{2}\left|\int f(x-y) \psi_{n}(y) d y\right|^{2} d x \leq \int\langle x\rangle^{2}\left(\int|f(x-y)|^{2} \psi_{n}(y) d y\right) d x, \\
& \leq 2 \int|f(x)|^{2} d x+2 \int\left(|x-y|^{2}+|y|^{2}\right)\left(\int|f(x-y)|^{2} \psi_{n}(y) d y\right) d x \\
& \leq 4 \int|f(x)|^{2} d x+2 \int|x|^{2}|f(x)|^{2} d x=2 \int\langle x\rangle^{2}|f(x)|^{2} d x .
\end{aligned}
$$

In particular we have:

$$
\int_{\mathbb{R}^{3}}\langle x\rangle\left(\left|\operatorname{Re} \varphi_{0}^{n}\right|^{2}+\left|\nabla \varphi_{0}^{n}\right|^{2}\right) d x \leq 2 \int_{\mathbb{R}^{3}}\langle x\rangle\left(\left|\operatorname{Re} \varphi_{0}\right|^{2}+\left|\nabla \varphi_{0}\right|^{2}\right) d x=\delta_{1} .
$$

In addition $\varphi_{0}^{n}$ is uniformly bounded in $H^{\frac{5}{4}+\epsilon}\left(\mathbb{R}^{3}\right)$ then taking $\delta_{1}$ small enough there exist some sequence $\varphi_{n}$ solution of the Gross-Pitaevskii equation via Theorem 1.2. Furthermore from Sect. 3.3, we have $\left|\psi_{n}\right| \geq c^{\prime \prime}>0$ uniformly in $n$ (because $\left(1+|\xi|^{\epsilon_{1}}\right) \widehat{\varphi_{0}^{n}}$ is uniformly bounded in $L^{1}$, this is due to the fact that $\widehat{\psi}_{n}$ is uniformly bounded in $L^{\infty}$ since $\psi_{n}$ is uniformly bounded in $L^{1}$ ).

Since $\psi_{n}$ is smooth, according to the previous paragraph $\left(\rho_{n}, u_{n}=\left(\left|1+\varphi_{n}\right|^{2}\right.\right.$, $2 \operatorname{Im}\left(\nabla \varphi_{n} \frac{1+\overline{\varphi_{n}}}{\left|1+\varphi_{n}\right|^{2}}\right)$ is a global weak solution of the system (1.1) with initial data $\left(\rho_{0}^{n}, u_{0}^{n}\right)$.

We recall now that:

$$
\begin{aligned}
& \rho_{n}(t, x)-1=2 \operatorname{Re}\left(\varphi_{n}\right)+\left|\varphi_{n}\right|^{2}, \\
& u_{n}(t, x)=\nabla \theta_{n}(t, x)=\operatorname{Im}\left(\left[\left(\frac{1+\overline{\varphi_{n}(t, x)}}{\left|1+\varphi_{n}(t, x)\right|^{2}}-1\right) \nabla \varphi_{n}(t, x)+\nabla \varphi_{n}(t, x)\right]\right) .
\end{aligned}
$$


Again using paraproduct laws we deduce easily ( since $\varphi_{n}$ is uniform bounded in $L_{T, x}^{\infty}$ ) that $\left(\rho_{n}-1\right)$ is uniformly bounded in $L_{T}^{\infty}\left(H^{\frac{5}{4}+\epsilon}\left(\mathbb{R}^{3}\right)\right)$ for any $T>0$. We observe now by using Propositions 2.3 and 2.5 that:

$$
\begin{aligned}
&\left\|u_{n}\right\|_{L_{T}^{\infty}\left(H^{2 \epsilon}\right)} \lesssim \|\left(\frac{1+\overline{\varphi_{n}(t, x)}}{\left|1+\varphi_{n}(t, x)\right|^{2}}-1\left\|_{L_{T}^{\infty}\left(L^{\infty}\right)}\right\| \varphi_{n} \|_{L_{T}^{\infty}\left(H^{\frac{5}{4}+\epsilon}\right)}\right. \\
&+\|\left(\frac{1+\overline{\varphi_{n}(t, x)}}{\left|1+\varphi_{n}(t, x)\right|^{2}}-1\left\|_{L_{T}^{\infty}\left(H^{\frac{5}{4}+\epsilon}\right)}\right\| \varphi_{n}\left\|_{L_{T}^{\infty}\left(H^{\frac{5}{4}+\epsilon}\right)}+\right\| \varphi_{n} \|_{L_{T}^{\infty}\left(H^{\frac{5}{4}+\epsilon}\right)}\right. \\
& \leq\left\|\varphi_{n}\right\|_{L_{T}^{\infty}\left(H^{\frac{5}{4}+\epsilon}\right)}\left(1+C_{2}\left(\left\|\varphi_{n}\right\|_{L_{T, x}^{\infty}},\left\|\frac{1}{\left|\psi_{n}\right|}\right\|_{L_{T, x}^{\infty}}\right)\left(1+\left\|\varphi_{n}\right\|_{L_{T}^{\infty}\left(H^{s}\right)}\right)\right) .
\end{aligned}
$$

We have proved that $u_{n}$ belongs uniformly to $L^{\infty}\left(H^{2 \epsilon}\left(\mathbb{R}^{3}\right)\right)$. From Sect. $3.2\left(\rho_{n}, \frac{1}{\rho_{n}}\right)$ is uniformly bounded in $L^{\infty}\left(\mathbb{R}^{+}, L^{\infty}\left(\mathbb{R}^{3}\right)\right)$. We observe now from Plancherel theorem that there exist $C, \epsilon>0$ such that:

$$
\begin{aligned}
\left\|\varphi_{n}-\varphi\right\|_{C\left([0, T], H^{\frac{5}{4}+\frac{\epsilon}{2}}\left(\mathbb{R}^{3}\right)\right)}^{2} \leq & \left\|\left(1-\widehat{\psi_{n}}\right) 1_{\{|\xi| \leq \epsilon n\}}\right\|_{L^{\infty}}^{2}\|\varphi\|_{C\left([0, T], H^{\frac{5}{4}+\frac{\epsilon}{2}}\left(\mathbb{R}^{3}\right)\right)}^{2} \\
& +C \int_{\{|\xi| \geq \epsilon n\}}\left(1+|\xi|^{2}\right)^{\frac{5}{4}+\frac{\epsilon}{2}}\left|\widehat{\varphi_{n}}(t, \xi)\right|^{2} d \xi .
\end{aligned}
$$

We deduce now since $\varphi_{n}$ is uniformly bounded in $L_{T}^{\infty}\left(H^{\frac{5}{4}+\epsilon}\left(\mathbb{R}^{3}\right)\right)$ that:

$$
\lim _{n \rightarrow+\infty}\left\|\varphi_{n}-\varphi\right\|_{C\left([0, T], H^{\frac{5}{4}+\frac{\epsilon}{2}}\left(\mathbb{R}^{3}\right)\right)}=0 .
$$

Using again paraproduct law we prove easily that:

$$
\begin{aligned}
& \lim _{n \rightarrow+\infty}\left\|\left(\rho_{n}-1\right)-(\rho-1)\right\|_{C\left([0, T], H^{\frac{5}{4}+\frac{\epsilon}{2}}\left(\mathbb{R}^{3}\right)\right)}=0, \\
& \lim _{n \rightarrow+\infty}\left\|u_{n}-u\right\|_{C\left([0, T], H^{\epsilon}\left(\mathbb{R}^{3}\right)\right)}=0 .
\end{aligned}
$$

It implies easily from Proposition 2.5 that $\left(\nabla \sqrt{\rho_{n}}\right)_{n \in \mathbb{N}}$ converges strongly in $C([0, T]$, $\left.H^{\frac{1}{4}+\frac{\epsilon}{2}}\left(\mathbb{R}^{3}\right)\right)$ by using Proposition 2.5 and the fact that $\left(\frac{1}{\rho^{n}}, \rho^{n}\right)$ is uniformly bounded in $L_{T}^{\infty}\left(L^{\infty}\right)$. In particular it yields the strong convergence of $\left(\nabla \sqrt{\rho_{n}}\right)_{n \in \mathbb{N}}$ in $L_{\text {loc }}^{2}\left(\mathbb{R}^{+} \times \mathbb{R}^{3}\right)$ to $\nabla \sqrt{\rho}$ which is sufficient to pass to the limit in the sense of distribution in the capillary terms. We proceed similarly in order to deal with the terms $\rho_{n} u_{n}=u_{n}+\left(\rho_{n}-1\right) u_{n}$ and $\rho_{n} u_{n} \times u_{n}=u_{n} \times u_{n}+\left(\rho_{n}-1\right) u_{n} \times u_{n}$. Indeed we know that $u_{n}$ converges strongly to $u$ in $C\left([0, T], L^{2+\alpha(\epsilon)}\right)$ with $\alpha(\epsilon)>0$ by Sobolev embedding. Furthermore since $\left(\rho_{n}-1\right)$ converges strongly in $C\left([0, T], L^{2}\right)$ and is uniformly bounded in $L_{T, x}^{\infty}$, it implies that $\left(\rho_{n}-1\right)$ converges strongly in any $C\left([0, T], L^{p}\right)$ with $2 \leq p<+\infty$. By Hölder inequality it achieves the proof of the existence of a global weak solution.

\subsection{Existence of global strong solution when $N \geq 3$}

In the previous section we have proved the existence of a global weak solution for the EulerKorteweg system (1.1) when $N=3$ under the assumption of smallness (1.16) and the fact that $\varphi_{0} \in H^{\frac{5}{4}+\epsilon}\left(\mathbb{R}^{3}\right)$. With additional assumption on $\varphi_{0} \in H^{\frac{N}{2}+1+\epsilon}$ we obtain new control on $q=\rho-1$ and $u$ since we have shown by using Proposition 3.12 that they belong respectively in $L_{l o c}^{\infty}\left(H^{\frac{N}{2}+1+\epsilon}\right) \cap L_{l o c}^{2}\left(B_{q, 2}^{\frac{N}{2}+1+\epsilon}\right)$ (with $\left.q=\frac{2 N}{N-2}\right)$ and in $L_{l o c}^{\infty}\left(H^{\frac{N}{2}+\epsilon}\right) \cap L_{l o c}^{2}\left(B_{q, 2}^{\frac{N}{2}+\epsilon}\right)$. 
Our main task is to prove now that under these controls the global weak solution that we have constructed is unique. Let us start by rewriting the system (1.1) using the change of unknown introduced by Benzoni et al. in [5]:

$$
\left\{\begin{array}{l}
\partial_{t} \ln \rho+u \cdot \nabla \ln \rho+\operatorname{div} u=0 \\
\partial_{t} z+u \cdot \nabla z+i \nabla z \cdot w+i \nabla \operatorname{div} z=\rho w
\end{array}\right.
$$

with:

$$
w=\nabla \ln \rho, L=\ln \rho, z=u+i w .
$$

The following proposition is proved in [5] (Proposition 3.3, p. 11) using a gauge method.

Proposition 3.3 Let $z$ be a $H^{s}$ solution with $s>0$ of:

$$
\left\{\begin{array}{l}
\partial_{t} \rho+\operatorname{div}(\rho v)=\rho g \\
\partial_{t} z+v \cdot \nabla z+i \nabla z \cdot w+i \nabla \operatorname{div} z=f
\end{array}\right.
$$

on $[0, T] \times \mathbb{R}^{N}$ with $w=\nabla \ln \rho$. Then the following estimates hold true for all $t \in[0, T]$ and $\alpha \in[0,1)$ :

$$
\begin{aligned}
\|z(t)\|_{H^{s}}^{2} \lesssim & \left\|z_{0}\right\|_{H^{s}}^{2}+\int_{0}^{t}\left(\|f\|_{H^{s}}\|z\|_{H^{s}}+A(\tau)\|z\|_{H^{s}}^{2}\right) d \tau \\
& +\|w(t)\|_{C^{-\alpha}}^{2}\|z(t)\|_{H^{s-1+\alpha}}^{2} .
\end{aligned}
$$

and:

$$
\|(\sqrt{\rho} z)(t)\|_{L^{2}}^{2} \lesssim\|\sqrt{\rho} z(0)\|_{L^{2}}^{2}+\int_{0}^{t}\|\sqrt{\rho} z\|_{L^{2}}\|\sqrt{\rho} f\|_{L^{2}} d \tau .
$$

with:

$$
A(t)=1+\|D z(t)\|_{L^{\infty}}+\|g(t)\|_{L^{\infty}} .
$$

Benzoni et al obtain the following corollary (see 4.2, p. 23 in [5]).

Corollary 3.4 Let $(L, z)$ satisfy the assumptions of Proposition 3.3 with $g=0$, then we have for $C>0$ :

$$
\|z\|_{L_{T}^{\infty}\left(H^{s}\right)} \lesssim e^{C \int_{0}^{T} A(\tau) d \tau}\left(1+\|w\|_{L_{T}^{\infty}\left(L^{\infty}\right)}^{\max (1, s)}\right)\left(\left\|z_{0}\right\|_{H^{s}}+\|f\|_{L_{T}^{1}\left(H^{s}\right)}\right) .
$$

Remark 14 Let us mention that if $z$ is irrotational, then we can extend the range of $s$ to $s>-\frac{N}{2}$ (see the remark 4.1, p. 24 of [5]).

In the spirit of Proposition 5.1, p. 29 of [5] we obtain the following proposition.

Proposition 3.5 Let $N \geq 3$. Let $\left(L_{1}=\ln \rho_{1}, z_{1}\right)$ and $\left(L_{2}=\ln \rho_{2}, z_{2}\right)$ be two solutions of (3.21) on $[0, T] \times \mathbb{R}^{N}$ in $\left(L^{\infty}\left(H^{s+1}\right) \cap L^{2}\left(B_{q, 2}^{s+1}\right)\right) \times\left(L^{\infty}\left(H^{s}\right) \cap L^{2}\left(B_{q, 2}^{s}\right)\right.$ with $\frac{N}{2}<s$ and $q=\frac{2 N}{N-2}$. Assume in addition that $L_{i}(i=1,2)$ is bounded in $L^{\infty}$. Let us denote $\delta L=L_{2}-L_{1}$ and $\delta z=z_{2}-z_{1}$. Then the following estimate hold true for all $t \in[0, T]$ with $0<s^{\prime}<\frac{N}{2}-1$ :

$$
\|(\delta L(t), \delta z(t))\|_{H^{s^{\prime}}} \lesssim\left(\|\delta L(0)\|_{H^{s^{\prime}}} \theta_{1}(t)+\|\delta z(0)\|_{H^{s^{\prime}}} \theta_{2}(t)\right) \theta_{3}(t),
$$

with $\theta_{1}, \theta_{2}$ and $\theta_{3}$ continuous positive functions. These functions depend on the following norms $\left\|q_{i}\right\|_{\left.L_{t}^{\infty}\left(H^{s+1}\right) \cap L_{t}^{2}\left(B_{q, 2}^{s+1}\right)\right)}$ and $\left\|u_{i}\right\|_{L_{t}^{\infty}\left(H^{s}\right) \cap L_{t}^{2}\left(B_{q, 2}^{s}\right)}$ with $i \in\{1,2\}$. 
Remark 15 Let us mention that this proposition improves Proposition 5.1 p 29 of [5] where $\left\|D u_{i}\right\|_{H^{s}}<\infty$ is required.

Proof The equation satisfied by $\delta z$ reads:

$$
\partial_{t} \delta z+u_{1} \cdot \nabla \delta z+i \nabla \delta z \cdot w_{1}+i \nabla \operatorname{div} \delta z=\nabla \delta \rho-(\delta u) \cdot \nabla z_{2}-i \nabla z_{2} \cdot \delta w
$$

with $\delta u=u_{2}-u_{1}, \delta \rho=\rho_{2}-\rho_{1}$ and $\delta w=w_{2}-w_{1}$. We observe that $\delta z$ solves an equation of type (3.22) since $\rho_{1}$ verifies the mass equation $\partial_{t} \rho_{1}+\operatorname{div}\left(\rho_{1} u_{1}\right)=0$ and $w_{1}=\nabla \ln \rho_{1}$ (we are in particular in the case $g=0$ ). Applying corollary 3.4 we can estimate $\delta z$ in $H^{s^{\prime}}$ with $s^{\prime}>0$ (that we will define later) as follows when $t \in[0, T]$ :

$$
\begin{array}{r}
\|\delta z(t)\|_{H^{s^{\prime}}} \lesssim \gamma(t) e^{C \int_{0}^{t}\left(1+\left\|D z_{1}\right\|_{L} \infty\right) d \tau}\left(\|\delta z(0)\|_{H^{s^{\prime}}}+\int_{0}^{t}\left(\|\nabla \delta \rho\|_{H^{s^{\prime}}}+\left\|\delta u \cdot \nabla z_{2}\right\|_{H^{s^{\prime}}}\right.\right. \\
\left.\left.+\left\|\nabla z_{2} \cdot \delta w\right\|_{H^{s^{\prime}}} d \tau\right)\right),
\end{array}
$$

with $\gamma(t)=1+\left\|w_{1}\right\|_{L_{t}^{\infty}\left(L^{\infty}\right)}^{\max \left(1, s^{\prime}\right)}$. Let us mention that in our case since $L_{1}$ is bounded in $L_{T}^{\infty}\left(H^{s+1}\right)$ with $s>\frac{N}{2}$ we deduce by Proposition 2.5 that $w_{1}$ is bounded in $L_{T}^{\infty}\left(L^{\infty}\right)$. Similarly $D z_{1}$ belongs to $L_{T}^{1}\left(L^{\infty}\right)$ by Besov embedding since $\nabla z_{1}$ is in $L_{T}^{2}\left(B_{\frac{2 N}{N-2}, 2}^{s-1}\right)$ and $s>\frac{N}{2}$.

It remains now to estimate the integrand in the right-hand side of (3.27). By Proposition 2.3 since we have $\frac{1}{2} \leq \frac{N-2}{2 N}+\frac{1}{\lambda} \leq 1$ with $\lambda=N \in[1,+\infty], 2 \leq N$ and $s^{\prime}+1<\frac{N}{2}$ then:

$$
\begin{aligned}
&\left\|T_{\delta u} \nabla z_{2}\right\|_{H^{s^{\prime}}} \lesssim\|\delta u\|_{H^{s^{\prime}}}\left\|\nabla z_{2}\right\|_{B^{\frac{N}{2}-1} \frac{N}{N-2}, \infty}, \\
&\left\|T_{\nabla z_{2}} \delta u\right\|_{H^{s^{\prime}}} \lesssim\left\|\nabla z_{2}\right\|_{L^{\infty}}\|\delta u\|_{H^{s^{\prime}}} .
\end{aligned}
$$

Similarly we have since $s^{\prime}+\frac{N-2}{2}=s^{\prime}+\frac{N}{2}-1>0$ then:

$$
\left\|R\left(\nabla z_{2}, \delta u\right)\right\|_{H^{s^{\prime}}} \lesssim\left\|\nabla z_{2}\right\|_{\substack{\frac{N}{2}-1 \\ \frac{2 N}{N-2}, \infty}}\|\delta u\|_{H^{s^{\prime}}}
$$

We deduce that if $0<s^{\prime}<\frac{N}{2}-1$ :

$$
\left\|\delta u \cdot \nabla z_{2}\right\|_{H^{s^{\prime}}}+\left\|\nabla z_{2} \cdot \delta w\right\|_{H^{s^{\prime}}} \lesssim\left\|D z_{2}\right\|_{L^{\infty} \cap B_{\frac{2 N}{N-2}, \infty}^{\frac{N}{2}-1}}\|\delta z\|_{H^{s^{\prime}}}
$$

Let us deal now with the term $\delta \rho$ and we set $\delta L=\ln \rho_{2}-\ln \rho_{1}$ with $L=\ln \rho$, we have then:

$$
\begin{aligned}
\|\nabla \delta \rho\|_{H^{s^{\prime}}} \leq\|\delta \rho\|_{H^{s^{\prime}+1}} & \lesssim \int_{0}^{1}\left\|\delta L \exp \left(L_{1}+\tau \delta L\right)\right\|_{H^{s^{\prime}+1}} d \tau \\
& \left.\lesssim \int_{0}^{1}\left\|\delta L\left(\exp \left(L_{1}+\tau \delta L\right)-1\right)\right\|_{H^{s^{\prime}+1}}+\|\delta L\|_{H^{s^{\prime}+1}}\right) d \tau
\end{aligned}
$$


Next for $\tau \in[0,1]$ we have using Proposition 2.3, 2.5, $s^{\prime}+1-\frac{N}{2}<0$ and the fact that $L_{1}=\ln \left(1+q_{1}\right), L_{2}=\ln \left(1+q_{2}\right) \in L^{\infty}$ :

$$
\begin{aligned}
& \left\|T_{\left(\exp \left(L_{1}+\tau \delta L\right)-1\right)} \delta L\right\|_{H^{s^{\prime}+1}} \lesssim\|\delta L\|_{H^{s^{\prime}+1}}\left\|\left(\exp \left(L_{1}+\tau \delta L\right)-1\right)\right\|_{L^{\infty}}, \\
& \left\|T_{\delta L}\left(\exp \left(L_{1}+\tau \delta L\right)-1\right)\right\|_{H^{s^{\prime}+1}} \lesssim\|\delta L\|_{B_{\infty, 2}^{s^{\prime}+1-\frac{N}{2}}}\left\|\left(\exp \left(L_{1}+\tau \delta L\right)-1\right)\right\|_{B_{2, \infty}^{\frac{N}{2}}} \\
& \lesssim\|\delta L\|_{H^{s^{\prime}+1}}\left\|\left(\exp \left(L_{1}+\tau \delta L\right)-1\right)\right\|_{B_{2, \infty}^{2}} \\
& \lesssim\|\delta L\|_{H^{s^{\prime}+1}} C\left(\left\|\left(\rho_{1}, \frac{1}{\rho_{1}}, \rho_{2}, \frac{1}{\rho_{2}}\right)\right\|_{L^{\infty}}\right)\left(\left\|L_{1}\right\|_{L^{\infty} \cap B_{2, \infty}^{\frac{N}{2}}}+\left\|L_{2}\right\|_{L^{\infty} \cap B_{2, \infty}^{\frac{N}{2}}}\right) \\
& \lesssim\|\delta L\|_{H^{s^{\prime}+1}} C^{\prime}\left(\left\|\left(\rho_{1}, \frac{1}{\rho_{1}}, \rho_{2}, \frac{1}{\rho_{2}}\right)\right\|_{L^{\infty}}\right)\left(\left\|q_{1}\right\|_{L^{\infty} \cap B_{2, \infty}^{\frac{N}{2}}}+\left\|q_{2}\right\|_{L^{\infty} \cap B_{2, \infty}^{\frac{N}{2}}}\right), \\
& \left.\| R\left(\exp \left(L_{1}+\tau \delta L\right)-1\right), \delta L\right)\left\|_{H^{s^{\prime}+1}} \lesssim\right\| \delta L\left\|_{H^{s^{\prime}+1}}\right\| \exp \left(L_{1}+\tau \delta L\right)-1 \|{ }_{B_{2, \infty}^{\frac{N}{2}}} \\
& \lesssim\|\delta L\|_{H^{s^{\prime}+1}} C_{1}^{\prime}\left(\left\|\left(\rho_{1}, \frac{1}{\rho_{1}}, \rho_{2}, \frac{1}{\rho_{2}}\right)\right\|_{L^{\infty}}\right)\left(\left\|q_{1}\right\|_{L^{\infty} \cap B_{2, \infty}^{\frac{N}{2}}}+\left\|q_{2}\right\|_{L^{\infty} \cap B_{2, \infty}^{\frac{N}{2}}}\right) .
\end{aligned}
$$

Plugging all these inequalities in (3.27), using the fact that:

$$
\|\delta L\|_{H^{s^{\prime}+1}} \leq\|\delta z\|_{H^{s^{\prime}}}+\|\delta L\|_{L^{2}} \leq\|\delta z\|_{H^{s^{\prime}}}+\|\delta L\|_{H^{s^{\prime}}}
$$

we have for $\left.s^{\prime} \in\right] 0, \frac{N}{2}-1[$ :

$$
\begin{aligned}
& \|\delta z(t)\|_{H^{s^{\prime}}} \lesssim \gamma(t) e^{C \int_{0}^{t}\left(1+\left\|D z_{1}\right\|_{L^{\infty}}\right) d \tau}\left(\|\delta z(0)\|_{H^{s^{\prime}}}\right. \\
& +\int_{0}^{t}\left(\|\delta L(\tau)\|_{H^{s^{\prime}}} C_{1}^{\prime}\left(\left\|\left(\rho_{1}, \frac{1}{\rho_{1}}, \rho_{2}, \frac{1}{\rho_{2}}\right)(\tau)\right\|_{L^{\infty}}\right)\left(\left\|q_{1}(\tau)\right\|_{L^{\infty} \cap B_{2, \infty}^{\frac{N}{2}}}+\left\|q_{2}(\tau)\right\|_{L^{\infty} \cap B_{2, \infty}^{\frac{N}{2}}}\right)\right.
\end{aligned}
$$

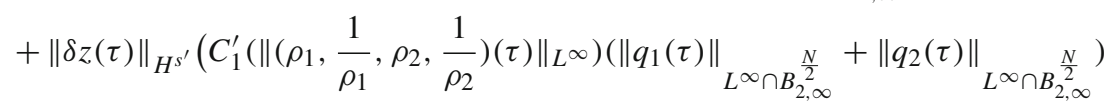

$$
\begin{aligned}
& \left.\left.\left.+\left\|D z_{2}(\tau)\right\|_{L^{\infty \cap B} \frac{\frac{N}{2}-1}{N-2}, \infty}^{\frac{2 N}{2}}\right)\right) d \tau\right) .
\end{aligned}
$$

Applying Gronwall's inequality, we end up with:

$$
\|\delta z(t)\|_{H^{s^{\prime}}} \lesssim \varphi(t)+\int_{0}^{t} \varphi(s) \psi(s) \exp \left(\int_{s}^{t} \psi(u) d u\right) d s,
$$

with for $s \in[0, t]$ :

$$
\begin{aligned}
\varphi(s)= & \gamma(s) e^{C \int_{0}^{s}\left(1+\left\|D z_{1}\right\|_{L}\right) d \tau} \\
& \times\left(\|\delta z(0)\|_{H^{s^{\prime}}}+\int_{0}^{s}\left(\| \delta L ( \tau ) \| _ { H ^ { s ^ { \prime } } } C _ { 1 } ^ { \prime } ( \| ( \rho _ { 1 } , \frac { 1 } { \rho _ { 1 } } , \rho _ { 2 } , \frac { 1 } { \rho _ { 2 } } ) ( \tau ) \| _ { L ^ { \infty } } ) \left(\left\|q_{1}(\tau)\right\|_{L^{\infty} \cap B_{2, \infty}^{\frac{N}{2}}}\right.\right.\right. \\
& \left.\left.\left.+\left\|q_{2}(\tau)\right\|_{L^{\infty} \cap B_{2, \infty}^{\frac{N}{2}}}\right)\right) d \tau\right) .
\end{aligned}
$$

and for $C>0$ : 


$$
\begin{aligned}
\psi(s)= & C \gamma(t) e^{C \int_{0}^{t}\left(1+\left\|D z_{1}\right\|_{L} \infty\right) d \tau} \\
& \times\left(C_{1}^{\prime}\left(\left\|\left(\rho_{1}, \frac{1}{\rho_{1}}, \rho_{2}, \frac{1}{\rho_{2}}\right)(s)\right\|_{L^{\infty}}\right)\left(\left\|q_{1}(s)\right\|_{L^{\infty} \cap B_{2, \infty}^{\frac{N}{2}}}+\left\|q_{2}(s)\right\|_{L^{\infty} \cap B_{2, \infty}^{\frac{N}{2}}}\right)\right. \\
& \left.+\left\|D z_{2}(s)\right\|_{L^{\infty} \cap B^{\frac{N}{2}-1}}\right) .
\end{aligned}
$$

In order to close the estimate we have to deal with the term $\|\delta L(\tau)\|_{H^{s^{\prime}}}$ which appears in the right-hand side of (3.31) (in the term $\varphi(t)$ ). In order to do this, we use the fact that $\delta L=\ln \rho_{2}-\ln \rho_{1}$ satisfies the following equation:

$$
\partial_{t} \delta L+u_{2} \cdot \nabla \delta L+\delta u \cdot \nabla L_{1}+\operatorname{div} \delta u=0,
$$

and:

$$
\partial_{t} \Delta_{l} \delta L+u_{2} \cdot \nabla \Delta_{l} \delta L+\Delta_{l}\left(\delta u \cdot \nabla L_{1}+\operatorname{div} \delta u\right)=\left[u_{2}^{j}, \Delta_{l}\right] \partial_{j} \delta L .
$$

Taking the $L^{2}$ inner product of the above equation with $\Delta_{l} \delta L$, performing several integration by parts and integrate in time we get:

$$
\begin{aligned}
\left\|\Delta_{l} \delta L(t)\right\|_{L^{2}}^{2} \lesssim & \left\|\Delta_{l} \delta L_{0}\right\|_{L^{2}}^{2}+\int_{0}^{t}\left(\left\|\Delta_{l}\left(\delta u \cdot \nabla L_{1}\right)(\tau)\right\|_{L^{2}}\left\|\Delta_{l} \delta L(\tau)\right\|_{L^{2}}\right. \\
+ & \left.\left\|\Delta_{l} \delta u\right\|_{L^{2}}\left\|\Delta_{l}(\delta w)(\tau)\right\|_{L^{2}}\right) d \tau+\int_{0}^{t}\left(\left\|\Delta_{l} \delta L(\tau)\right\|_{L^{2}}\left\|R_{l}(\tau)\right\|_{L^{2}}\right. \\
& +\left\|\operatorname{div} u_{2}(\tau)\right\|_{\left.L^{\infty}\left\|\Delta_{l} \delta L(\tau)\right\|_{L^{2}}^{2}\right) d \tau}
\end{aligned}
$$

We have set $R_{l}=\left[u_{2}^{j}, \Delta_{l}\right] \partial_{j} \delta L$. Using Lemma 2.6 (since $s^{\prime}<\frac{N}{\frac{2 N}{N-2}}=\frac{N}{2}-1$ ), multiplying the previous equation by $2^{2 l s^{\prime}}$ and summing we have:

$$
\begin{aligned}
\|\delta L(t)\|_{H^{s^{\prime}}}^{2} \lesssim & \left\|\delta L_{0}\right\|_{H^{s^{\prime}}}^{2}+\int_{0}^{t}\left\|\nabla u_{2}(\tau)\right\|_{L^{\infty} \cap B_{\frac{2 N}{N-2}, \infty}^{\frac{N}{2}-1}}\|\delta L(\tau)\|_{H^{s^{\prime}}}^{2} d \tau \\
& +\int_{0}^{t}\left(\left\|\delta u \cdot \nabla L_{1}(\tau)\right\|_{H^{s^{\prime}}}\|\delta L(\tau)\|_{H^{s^{\prime}}}+\|\delta u\|_{H^{s^{\prime}}}\|\delta w(\tau)\|_{H^{s^{\prime}}}\right) d \tau .
\end{aligned}
$$

Next by using Proposition 2.3 we have since $s^{\prime}<\frac{N}{2}-1$ :

$$
\left\|\delta u \cdot \nabla L_{1}\right\|_{H^{s^{\prime}}} \lesssim\|\delta u\|_{H^{s^{\prime}}}\left\|\nabla L_{1}\right\|_{L^{\infty} \cap B} \frac{\frac{N}{N-2}-1}{\frac{2 N}{N-2}, \infty} .
$$

Plugging this inequalities in (3.33) and using the fact that $\|\delta u\|_{H^{s^{\prime}}}\|\delta L\|_{H^{s^{\prime}}} \leq \frac{1}{2}\left(\|\delta z\|_{H^{s^{\prime}}}^{2}+\right.$ $\|\delta L\|_{H^{s^{\prime}}}^{2}$ ) we have:

$$
\begin{aligned}
\|\delta L(t)\|_{H^{s^{\prime}}}^{2} \lesssim & \left\|\delta L_{0}\right\|_{H^{s^{\prime}}}^{2}+\int_{0}^{t}\left(\left\|\nabla u_{2}(\tau)\right\|_{L^{\infty} \cap B_{\frac{2 N}{N-2}, \infty}^{\frac{N}{2}-1}}+\left\|\nabla L_{1}(\tau)\right\|_{L^{\infty} \cap B_{\frac{2 N}{N-2}, \infty}^{\frac{N}{2}-1}}\right)\|\delta L(\tau)\|_{H^{s^{\prime}}}^{2} d \tau \\
& +\int_{0}^{t}\left(1+\left\|\nabla L_{1}(\tau)\right\|_{L^{\infty} \cap B_{\frac{2 N}{N-2}, \infty}^{\frac{N}{2}-1}}\right)\|\delta z(\tau)\|_{H^{s^{\prime}}}^{2} d \tau .
\end{aligned}
$$

Using Gronwall's lemma we obtain:

$$
\|\delta L(t)\|_{H^{s^{\prime}}}^{2} \lesssim \varphi_{1}(t)+\int_{0}^{t} \varphi_{1}(s) \psi(s) \exp \left(\int_{s}^{t} \psi_{1}(u) d u\right) d s,
$$


with for $s \in[0, t]$ :

$$
\varphi_{1}(s)=\left\|\delta L_{0}\right\|_{H^{s^{\prime}}}^{2}+\int_{0}^{t}\left(1+\left\|\nabla L_{1}(\tau)\right\|_{L^{\infty \cap B} \frac{\frac{N N}{N-2}, \infty}{\frac{N}{2}-1}}\right)\|\delta z(\tau)\|_{H^{s^{\prime}}}^{2} d \tau
$$

and for $C>0$ :

$$
\psi_{1}(s)=C(\left\|\nabla u_{2}(s)\right\|_{L^{\infty} \cap B^{\frac{N}{N-2}}, \infty}+\left\|\nabla L_{1}(s)\right\| \underbrace{}_{L^{\infty} \cap B_{\frac{2 N}{N-2}, \infty}^{\frac{N}{2}-1}})
$$

It implies that:

$$
\|\delta L(t)\|_{H^{s^{\prime}}}^{2} \lesssim \varphi_{2}(t)\left(1+\int_{0}^{t} \psi_{1}(s) \exp \left(\int_{s}^{t} \psi_{1}(u) d u\right) d s\right),
$$

with:

$$
\varphi_{2}(t)=\left\|\delta L_{0}\right\|_{H^{s^{\prime}}}^{2}+\|\delta z\|_{L_{t}^{\infty}\left(H^{s^{\prime}}\right)}^{2} \int_{0}^{t}\left(1+\left\|\nabla L_{1}(\tau)\right\|_{L^{\infty} \cap B_{\frac{2 N}{N-2}, \infty}^{\frac{N}{2}-1}}\right) d \tau
$$

Taking now the $L_{t}^{\infty}$ norm of (3.31) and plugging it in (3.36), we have:

$$
\begin{aligned}
\|\delta L(t)\|_{H^{s^{\prime}}}^{2} \lesssim\left(\left\|\delta L_{0}\right\|_{H^{s^{\prime}}}^{2}+(\varphi(t)\right. & \left.\left.\left(1+\int_{0}^{t} \psi(s) \exp \left(\int_{s}^{t} \psi(u) d u\right) d s\right)\right)^{2}\right) \\
& \times\left(1+\int_{0}^{t} \psi_{1}(s) \exp \left(\int_{s}^{t} \psi_{1}(u) d u\right) d s\right) .
\end{aligned}
$$

From Hölder inequality and Young inequality, we deduce that

$$
\begin{aligned}
& \varphi^{2}(t) \leq \gamma^{2}(t) e^{2 C \int_{0}^{t}\left(1+\left\|D z_{1}\right\|_{L} \infty\right) d \tau} \times\left(2\|\delta z(0)\|_{H^{s^{\prime}}}^{2}+2 t \int_{0}^{t}\left(\|\delta L(\tau)\|_{H^{s^{\prime}}}^{2}\right.\right. \\
& \times\left(C_{1}^{\prime}\left(\left\|\left(\rho_{1}, \frac{1}{\rho_{1}}, \rho_{2}, \frac{1}{\rho_{2}}\right)(\tau)\right\|_{L^{\infty}}\right)\right)^{2}\left(\left\|q_{1}(\tau)\right\|_{L^{\infty} \cap B_{2, \infty}}{ }_{\frac{N}{2}}\right. \\
& \left.\left.\left.+\left\|q_{2}(\tau)\right\|_{L^{\infty} \cap B_{2, \infty}^{\frac{N}{2}}}\right)^{2}\right) d \tau\right) .
\end{aligned}
$$

It gives:

$$
\begin{aligned}
\|\delta L(t)\|_{H^{s^{\prime}}}^{2} \lesssim & \left(\left\|\delta L_{0}\right\|_{H^{s^{\prime}}}^{2}+\psi_{4}(t)\left(\|\delta z(0)\|_{H^{s^{\prime}}}^{2}+t \int_{0}^{t}\|\delta L(\tau)\|_{H^{s^{\prime}}}^{2} \psi_{3}(\tau) d \tau\right)\right. \\
& \left.\left.\times\left(1+\int_{0}^{t} \psi(s) \exp \left(\int_{s}^{t} \psi(u) d u\right) d s\right)\right)^{2}\right) \\
& \times\left(1+\int_{0}^{t} \psi_{1}(s) \exp \left(\int_{s}^{t} \psi_{1}(u) d u\right) d s\right) .
\end{aligned}
$$

with:

$$
\begin{aligned}
& \psi_{3}(t)=\left(C_{1}^{\prime}\left(\left\|\left(\rho_{1}, \frac{1}{\rho_{1}}, \rho_{2}, \frac{1}{\rho_{2}}\right)(\tau)\right\|_{L^{\infty}}\right)\right)^{2}\left(\left\|q_{1}(\tau)\right\|_{L^{\infty} \cap B_{2, \infty}^{\frac{N}{2}}}+\left\|q_{2}(\tau)\right\|_{L^{\infty} \cap B_{2, \infty}}{ }^{\frac{N}{2}}\right)^{2} \\
& \psi_{4}(t)=\gamma^{2}(t) e^{2 C \int_{0}^{t}\left(1+\left\|D z_{1}\right\|_{L}\right) d \tau}
\end{aligned}
$$

Using again Gronwall lemma it yields:

$$
\|\delta L(t)\|_{H^{s^{\prime}}}^{2} \lesssim\left(\|\delta L(0)\|_{H^{s^{\prime}}}^{2} \psi_{7}(t)+\|\delta z(0)\|_{H^{s^{\prime}}}^{2} \psi_{8}(t)\right) \psi_{6}(t),
$$


with $\psi_{6}, \psi_{7}, \psi_{8}$ continuous positive functions in time. It is important to observe that all the quantities $\int_{0}^{t} \psi(\tau) d \tau, \int_{0}^{t} \psi_{1}(\tau) d \tau, \int \psi_{3}(\tau) d \tau, \psi_{4}(t)$ are locally bounded in time. This is due to the fact that $\nabla u_{1}, \nabla u_{2}$ are in $L_{t}^{2}\left(B_{\frac{2 N}{N-2}, 2}^{\frac{N}{2}-1} \cap L^{\infty}\right)$ (and in particular is also $L_{l o c}^{1}\left(B_{\frac{2 N}{N-2}, 2}^{\frac{N}{2}-1} \cap L^{\infty}\right)$ ) and $q_{1}, q_{2}$ are in $L_{t}^{2}\left(B_{\frac{2 N}{N-2}, 2}^{\frac{N}{2}}\right) \cap L^{\infty}\left(B_{2,2}^{\frac{N}{2}+1}\right)$.

Finally, plugging (3.39) in (3.31) yields the desired inequality.

\section{Lipschitz control on the velocity $u$}

We recall that $\varphi$ belongs to $L_{l o c}^{2}\left(B_{6,2}^{s}\left(\mathbb{R}^{3}\right)\right.$ ) (with $s>\frac{3}{2}+1+\epsilon$ ) by propagation of the regularity for an initial data in $H^{s}$. From Proposition 3.12 we deduce that $u$ belongs to $L_{l o c}^{2}\left(B_{6,2}^{s-1}\right)$ for any $T>0$ and $\nabla u \in L_{T}^{2}\left(B_{6,2}^{s-2}\right)$. From Sobolev embedding we have obtained that $\nabla u$ is in $L_{T}^{2}\left(L^{\infty}\left(\mathbb{R}^{3}\right)\right)$ for any $T>0$ since $\frac{1}{6}-\frac{s-2}{3}<0$. Using Proposition 3.5 we conclude that the solution $(\rho, u)$ is unique.

\section{Proof of Theorem 1.3: global well-posedness for $N \geq 4$}

\subsection{A subcritical version of Theorem 1.1}

We prove in this section a simpler version of Theorem 1.1:

Proposition 4.1 Let $s>\frac{N}{2}-1, N \geq 4,1 / q=1 / 2-1 / N$. If $\left\|U^{-1} V \varphi_{0}\right\|_{H^{s}}<\varepsilon$, $\varepsilon$ small enough then the solution of $(G P)(1.8)$ is global and remains small:

$$
\left\|U^{-1} V \varphi\right\|_{L_{t}^{\infty} H^{s} \cap L_{t}^{2} B_{q, 2}^{s}} \lesssim\left\|U^{-1} V \varphi_{0}\right\|_{H^{s}} .
$$

Furthermore, $e^{i t H} U^{-1} V \varphi(t)$ converges in $H^{s}$ as $t \rightarrow \infty$.

As we work in subcritical settings both in term of regularity $\left(s>\frac{N}{2}-1\right)$ and scattering (in dimension 4 the Strauss exponent is $\simeq 1.78$ so that quadratic nonlinearities are not an issue), the only difficulty comes from the "diagonalization" : if $w=U^{-1} \varphi_{1}+i \varphi_{2}:=U^{-1} V \varphi$, then $w$ is solution of

$$
\begin{aligned}
i \partial_{t} w-H w & =\left(3 \varphi_{1}^{2}+\varphi_{2}^{2}+|\varphi|^{2} \varphi_{1}\right)+i U^{-1}\left(2 \varphi_{1} \varphi_{2}+|\varphi|^{2} \varphi_{2}\right) \\
& =\left(3\left(U w_{1}\right)^{2}+w_{2}^{2}+|V w|^{2} U v_{1}\right)+i U^{-1}\left(2 U w_{1} w_{2}+|V w|^{2} v_{2}\right) .
\end{aligned}
$$

and a singular multiplier $U^{-1}$ is present in the imaginary part of the nonlinearity. The remedy is a normal form introduced in [27] (then refined in [28]): the new variable

$$
Z=w+\frac{U^{-1}}{2-\Delta}|\varphi|^{2}:=w+\frac{1}{\langle\nabla\rangle^{2}}|\varphi|^{2}=U^{-1} \varphi_{1}+\frac{U^{-1}}{\langle\nabla\rangle^{2}}|\varphi|^{2}+i \varphi_{2},
$$

satisfies the following equation :

$$
\begin{aligned}
i \partial_{t} Z-H Z & =i \partial_{t} w-H w+\frac{2 i}{\langle\nabla\rangle^{2}} \operatorname{Re}\left(\bar{\varphi} \partial_{t} \varphi\right)-\frac{H}{\langle\nabla\rangle^{2}}|\varphi|^{2} \\
& =2 \varphi_{1}^{2}+|\varphi|^{2} \varphi_{1}-i \frac{U^{-1} \operatorname{div}}{\langle\nabla\rangle^{2}}\left[4 \varphi_{1} \nabla \varphi_{2}+\nabla\left(|\varphi|^{2} \varphi_{2}\right)\right]:=P(\varphi) .
\end{aligned}
$$


We see that this change of variable desingularizes the imaginary part since $U^{-1}$ div is a bounded operator on $L^{p}, 1<p<\infty$. Obviously, it remains necessary to check that the map $w \rightarrow Z$ is invertible in our functional settings.

Functional spaces Set $b:=\frac{1}{q}=\frac{1}{2}-\frac{1}{N}, \frac{1}{p}=\frac{1}{2}-\frac{1}{2 N}$ so that $(2, q),(4, p)$ are admissible Strichartz pairs. For $s>N / 2-1$ we define the space

$$
X=L^{\infty}\left(\mathbb{R}^{+}, H^{s}\left(\mathbb{R}^{N}\right)\right) \cap L^{2}\left(\mathbb{R}^{+}, B_{q, 2}^{s}\left(\mathbb{R}^{N}\right)\right) .
$$

In particular we note that by interpolation :

$$
\|u\|_{L_{t}^{4}\left(B_{p, 2}^{s}\right)} \lesssim\|u\|_{X}
$$

Mapping $w \rightarrow Z=w+U^{-1}\langle\nabla\rangle^{-2}\left|U V^{-1} w\right|^{2}$ For $\frac{1}{\widetilde{r}}=\frac{1}{r}+\frac{1}{N}<1, s>0$ according to the (dual) Sobolev embeddings

$$
\left\|U^{-1} f\right\|_{B_{r, 2}^{s}} \lesssim\left\|S_{0} U^{-1} f\right\|_{L^{r}}+\left\|\left(1-S_{0}\right) f\right\|_{B_{r, 2}^{s}} \lesssim\|f\|_{W^{1, \tilde{r}}}+\|f\|_{B_{\widetilde{r}, 2}^{s+1}} \lesssim\|f\|_{B_{\widetilde{r}, 2}^{s+1}}
$$

For $s>N / 2-1 \geq 1, \frac{1}{q^{\prime}}=\frac{1}{2}+\frac{1}{N}$, the product estimates from Proposition 2.3 yield

$$
\begin{gathered}
\left\|U^{-1}\langle\nabla\rangle^{-2}|f|^{2}\right\|_{H^{s}} \lesssim\left\||f|^{2}\right\|_{B_{q^{\prime}, 2}^{s-1}} \lesssim\|f\|_{H^{s-1}}\|f\|_{B_{N, 1}^{0}} \lesssim\|f\|_{H^{s}}^{2}, \\
\left\|U^{-1}\langle\nabla\rangle^{-2}|f|^{2}\right\|_{B_{q, 2}^{s}} \lesssim\|f\|_{H^{s}}\|f\|_{B_{q, 1}^{N / 2-1}} \lesssim\|f\|_{H^{s}}\|f\|_{B_{q, 2}^{s}} .
\end{gathered}
$$

From a fixed point argument, the map $w \rightarrow w+U^{-1}\langle\nabla\rangle^{-2}\left|U V^{-1} w\right|^{2}$ is Lipschitz with Lipschitz inverse $H^{s} \rightarrow H^{s}$ on a neighborhood of 0 . In particular for some $\delta>0$,

$$
\|Z\|_{H^{s}}<\delta \Rightarrow\|Z\|_{H^{s}} \sim\left\|U^{-1} V \varphi\right\|_{H^{s}},
$$

and provided $\|Z\|_{X}$ is small enough

$$
\|Z\|_{X} \sim\left\|U^{-1} V \varphi\right\|_{X} \gtrsim\|\varphi\|_{X} .
$$

Fixed point argument (sketch of) Scattering for $Z$ is equivalent to solve

$$
Z(t)=e^{-i t H} Z_{0}-i \int_{0}^{t} e^{-i(t-\tau) H} P(\varphi(\tau)) d \tau .
$$

From the Strichartz estimates of Proposition 2.17, we have

$$
\left\|e^{-i t H} Z_{0}-i \int_{0}^{t} e^{-i(t-\tau) H} P(\varphi(\tau)) d \tau\right\|_{X} \lesssim\left\|Z_{0}\right\|_{H^{s}}+\|P(\varphi)\|_{L^{2} B_{q^{\prime}, 2}^{s}},
$$

and we first check $\|P(\varphi)\|_{L^{2} B_{q^{\prime}}^{s}}\|\lesssim\| Z\left\|_{X}^{2}+\right\| Z \|_{X}^{3}$. For example, the term $\varphi_{1}^{2}$ can be estimated as follows

$$
\left\|\varphi_{1}^{2}\right\|_{L^{2} B_{q^{\prime}, 2}^{s}} \lesssim\left\|\varphi_{1}\right\|_{L^{\infty} H^{s}}\left\|\varphi_{1}\right\|_{L^{2} B_{q, 2}^{s}} \leq\left\|\varphi_{1}\right\|_{X}^{2} \lesssim\|Z\|_{X}^{2}
$$

The cubic term $U^{-1} \Delta\langle\nabla\rangle^{-2}\left(|\varphi|^{2} \varphi_{2}\right)$ is handled thanks to the embedding (4.2) (note that the multiplier $U^{-1} \Delta\langle\nabla\rangle^{-2}$ is not singular at $\xi=0$ ) 


$$
\begin{aligned}
\left\|U^{-1} \Delta\langle\nabla\rangle^{-2}\left(|\varphi|^{2} \varphi_{2}\right)\right\|_{L^{2} B_{q^{\prime}, 2}^{s}} \lesssim\left\||\varphi|^{2} \varphi_{2}\right\|_{L^{2} B_{q^{\prime}, 2}^{s}} & \lesssim\left\||\varphi|^{2}\right\|_{L^{2} H^{s}}\|\varphi\|_{L^{\infty} H^{s}} \\
& \lesssim\|\varphi\|_{L^{4} B_{p, 2}^{s}}^{2}\|\varphi\|_{L^{\infty} H^{s}} \\
& \lesssim\|Z\|_{X}^{3} .
\end{aligned}
$$

The other terms can be dealt with similarly, this gives

$$
\left\|e^{-i t H} Z_{0}-i \int_{0}^{t} e^{-i(t-\tau) H} P(\varphi(\tau)) d \tau\right\|_{X} \leq\left\|Z_{0}\right\|_{H^{s}}+\|Z\|_{X}^{2}+\|Z\|_{X}^{3} .
$$

Contractivity can be obtained by a similar argument since $P(\varphi)$ is essentially polynomial, and the fixed point theorem can be applied to obtain a unique global solution. The convergence of $e^{i t H} w$ follows from the convergence of $\int_{0}^{\infty} e^{i \tau H} P(\varphi) d \tau$ in $H^{s}$.

\section{2 $L^{\infty}$ bounds}

We follow the same plan as for Theorem 1.4.

Global control of $\|\varphi\|_{L^{\infty}}$

As a first step we prove time decay for $Z$, the global solution of (4.1)

$$
i \partial_{t} Z-H Z=2 \varphi_{1}^{2}+|\varphi|^{2} \varphi_{1}-i \frac{U^{-1} \operatorname{div}}{\langle\nabla\rangle^{2}}\left[4 \varphi_{1} \nabla \varphi_{2}+\nabla\left(|\varphi|^{2} \varphi_{2}\right)\right]=P(\varphi) .
$$

We set $1 / q=1 / 2-2 /(3 N), 1 / a^{\prime}=1 / 2+1 /(3 N), s>N / 2-1$. We have the following composition estimates (similar to (4.3),(4.4)):

$$
\begin{aligned}
& \left\|U^{-1}\langle\nabla\rangle^{-2}|\varphi|^{2}\right\|_{H^{s}} \lesssim\|\varphi\|_{H^{s}}^{2}, \\
& \left\|U^{-1}\langle\nabla\rangle^{-2}|\varphi|^{2}\right\|_{B_{a^{\prime}, 2}^{s}} \lesssim\left\|\varphi^{2}\right\|_{B_{6 N /(3 N+8), 2}^{s-1}} \lesssim\|\varphi\|_{H^{s-1}}\|\varphi\|_{B_{3 N / 4,1}^{0}} \lesssim\|\varphi\|_{H^{s}}^{2}, \\
& \left\|U^{-1}\langle\nabla\rangle^{-2}|\varphi|^{2}\right\|_{B_{q, 2}^{s}} \sim\|\varphi\|_{H^{s}}\|\varphi\|_{B_{q, 2}^{s}} .
\end{aligned}
$$

Using a fixed point argument, we deduce that for $\|Z\|_{H^{s} \cap B_{a^{\prime}, 2}^{s}}<<1$ resp. $\|Z\|_{H^{s} \cap B_{q, 2}^{s}}<<1$,

$$
\begin{aligned}
&\|Z\|_{H^{s} \cap B_{a^{\prime}, 2}^{s}} \sim\left\|U^{-1} V \varphi\right\|_{H^{s} \cap B_{a^{\prime}, 2}^{s}} \gtrsim\|\varphi\|_{H^{s} \cap B_{a^{\prime}, 2}^{s}}, \\
& \text { resp. }\|Z\|_{H^{s} \cap B_{q, 2}^{s,}} \sim\left\|U^{-1} V \varphi\right\|_{H^{s} \cap B_{q, 2}^{s}} \gtrsim\|\varphi\|_{H^{s} \cap B_{q, 2}^{s}}, \\
&\|Z\|_{B_{q, 2}^{s}} \sim\left\|U^{-1} V \varphi\right\|_{B_{q, 2}^{s}} .
\end{aligned}
$$

Proposition 4.2 Let $s>N / 2-1,1 / a^{\prime}=1 / 2+1 /(3 N), 1 / q=1 / 2-2 /(3 N), Z_{0} \in H^{s}$, $Z \in L^{\infty}\left(H^{s}\right) \cap L^{2}\left(B_{\frac{2 N}{N-2}, 2}^{s}\right)$ be the global solution of (4.1).

There exists $\varepsilon_{0}>0$ such that if $Z_{0} \in B_{a^{\prime}, 2}^{s+1 / 3}$, with $\left\|Z_{0}\right\|_{B_{a^{\prime}, 2}^{s+1 / 3} \cap H^{s}} \leq \varepsilon_{0}$, then

$$
\sup _{t \geq 0} t^{1 / 3}\|Z(t)\|_{B_{q, 2}^{s}} \lesssim\left\|Z_{0}\right\|_{B_{a^{\prime}, 2}^{s+1 / 3}}
$$

Proof We set $m(t)=\sup _{0 \leq \tau \leq t} \tau^{1 / 3}\|Z\|_{B_{q, 2}^{s}}$. The embedding $B_{a, 2}^{s+1 / 3} \hookrightarrow B_{q, 2}^{s}$ and the dispersion estimate give:

$$
\left\|e^{-i t H} Z_{0}\right\|_{B_{q, 2}^{s}} \lesssim\left\|e^{-i t H} Z_{0}\right\|_{B_{a, 2}^{s+1 / 3}} \lesssim \frac{1}{t^{N(1 / 2-1 / a)}}\left\|Z_{0}\right\|_{B_{a^{\prime}, 2}^{s+1 / 3}}=\frac{\left\|Z_{0}\right\|_{B_{a^{\prime}, 2}^{s+3}}}{t^{1 / 3}}
$$


Therefore it is only a matter of bounding the Duhamel term. Using Minkowski's inequality and the dispersion estimate (see Lemma 2.9) with $\theta=0, \sigma=\frac{2}{3 N}$ ) we obtain

$$
\left\|\int_{0}^{t} e^{i(t-\tau) H} P(\varphi) d \tau\right\|_{B_{q, 2}^{s}} \lesssim \int_{0}^{t} \frac{\|P(\varphi)(\tau, \cdot)\|_{B_{q^{\prime}, 2}^{s}}}{(t-\tau)^{2 / 3}} d \tau
$$

It remains to estimate $\|P(\varphi)(\tau, \cdot)\|_{B_{q^{\prime}, 2}^{s}}$. Arguing as in Sect. 4.1 it is sufficient to estimate $\left\|\varphi^{2}\right\|_{B_{q^{\prime}, 2}^{s}}+\left\|\varphi^{3}\right\|_{B_{q^{\prime}, 2}^{s}}$, this will be done by using paraproduct laws in Besov spaces. For quadratic terms, Proposition 2.3 with $q \leq \lambda=\frac{3 N}{4}$ gives since $s>\frac{N}{2}-2$ :

$$
\Rightarrow\left\|\varphi^{2}\right\|_{B_{q^{\prime}, 2}^{s}} \lesssim\|\varphi\|_{B_{q, 2}^{s}}\|\varphi\|_{B_{q, 1}^{\frac{N}{2}-2}} \lesssim\|\varphi\|_{B_{q, 2}^{s}}^{2} .
$$

For cubic terms we observe that $s+s+N\left(1 / q^{\prime}-1 / q-1 / 2-1 /(3 N)\right)=2 s+1-N / 2>s$, thus Proposition 2.3 gives

$$
\left\|\varphi^{2} \varphi\right\|_{B_{q^{\prime}, 2}^{s}} \lesssim\left\|\varphi^{2}\right\|_{B_{6 N /(3 N+2), 2}^{s}}\|\varphi\|_{B_{q, 2}^{s}} .
$$

Using again Proposition 2.3 we get

$$
\left\|\varphi^{2}\right\|_{B_{6 N /(3 N+2), 2}^{s}} \lesssim\|\varphi\|_{H^{s}}\|\varphi\|_{B_{q, 2}^{s}} \Rightarrow\left\|\varphi^{3}\right\|_{B_{q^{\prime}, 2}^{s}} \lesssim\|\varphi\|_{H^{s}}\|\varphi\|_{B_{q, 2}^{s}}^{2} .
$$

Plugging the quadratic and cubic estimate in (4.9) yield

$$
\begin{aligned}
& \left\|\int_{0}^{t} e^{i(t-\tau) H} P(\varphi)(\tau, \cdot) d s\right\|_{B_{q, 2}^{s}} \lesssim \int_{0}^{t} \frac{m(\tau)^{2}}{(t-\tau)^{2 / 3} \tau^{2 / 3}} d \tau \leq \frac{m(t)^{2}}{t^{1 / 3}} \int_{0}^{1} \frac{1}{(1-\tau)^{2 / 3} \tau^{2 / 3}} d \tau \\
& \quad \lesssim \frac{m(t)^{2}}{t^{1 / 3}}
\end{aligned}
$$

Using the Duhamel formula $m(t) \leq C\left\|Z_{0}\right\|_{B_{a^{\prime}, 2}^{s+1 / 3}}+C m(t)^{2}$, and from a bootstrap argument $\sup _{t} m(t) \lesssim\left\|Z_{0}\right\|_{B_{a^{\prime}, 2}^{s+1 / 3}}$.

Proposition 4.3 For $s>N / 2-1 / 4,1 / a=1 / 2-1 /(3 N)$, if $U^{-1} V \varphi_{0} \in H^{s} \cap B_{a^{\prime}, 2}^{s}$ there exists $\varepsilon_{0}$ such that

$$
\left\|U^{-1} V \varphi_{0}\right\|_{H^{s} \cap B_{a^{\prime}, 2}^{s}} \leq \varepsilon_{0} \text { and }\left\|e^{-i t \Delta} \varphi_{0}\right\|_{L^{\infty}\left([0,1] \times \mathbb{R}^{N}\right)} \leq 1 / 4 \Rightarrow\|\varphi\|_{L^{\infty}\left(\mathbb{R}^{+} \times \mathbb{R}^{N}\right)} \leq 1 / 2 .
$$

Proof If $\left\|U^{-1} V \varphi_{0}\right\|_{H^{s} \cap B_{a^{\prime}, 2}^{s}}<1$, from Proposition 4.2, $\|Z(t)\|_{B_{q, 2}^{s-1 / 3}} \lesssim \| U^{-1}$ $V \varphi_{0} \|_{B_{a^{\prime}, 2}^{s}} / t^{1 / 3}$. Using then (4.7) we get

$$
\forall t \geq 1,\|\varphi(t)\|_{L^{\infty}} \lesssim\|\varphi\|_{B_{q, 2}^{s-1 / 3}} \lesssim\|Z\|_{B_{q, 2}^{s-1 / 3}}<<1 .
$$

The bound on $\|\varphi\|_{L^{\infty}\left([0,1] \times \mathbb{R}^{N}\right)}$ is then obtained thanks to Theorem 3.1 :

$$
\begin{aligned}
\|\varphi\|_{L^{\infty}\left([0,1] \times \mathbb{R}^{N}\right)} & \leq\left\|e^{-i t \Delta} \varphi_{0}\right\|_{L^{\infty}\left([0,1] \times \mathbb{R}^{N}\right)}+C\left\|\varphi_{0}\right\|_{H^{s}}+C\left\|\int_{0}^{t} e^{i(t-s) \Delta} F(\varphi) d s\right\|_{H^{s+1 / 2}} \\
& \leq \frac{1}{4}+C \varepsilon_{0} .
\end{aligned}
$$

\section{End of the Proof of Theorem 1.3}

Proposition 4.3 provides the existence of a solution $\varphi$ to (1.8) such that $1+\varphi$ is bounded away from 0 . The rest of the proof is the same as for $N=3$, see paragraphs 3.5 and 3.6. 
Acknowledgements Corentin Audiard has been partially funded by the ANR project BoND ANR-13-BS010009-01. Boris Haspot has been partially funded by the ANR project INFAMIE ANR-15-CE40-0011.

\section{Appendix A: Sketch of proof of Theorem 1.2 when $N=3$}

For comfort of the reader, we outline in this appendix the main ideas of the (fairly long and technical) Proof of Theorem 1.2. For a complex number $f$, we use the non-standard notation:

$$
f^{+}=f, f^{-}=\bar{f} .
$$

\section{A.1 Space time resonances}

Let us consider the general nonlinear Schrödinger equation:

$$
i \partial_{t} u+\Delta u=f(u, \bar{u}), x \in \mathbb{R}^{d} .
$$

For $N=3$, the decay is not strong enough to obtain scattering with an argument similar to the one of Sect. 4.1, see also our discussion on the so-called Strauss exponent in the introduction. Well-posedness for small data in weighted spaces was obtained by Hayashi and Naumkin [31] in the case $f(u)=\lambda u^{2}+\mu \bar{u}^{2}$, while no global well-posedness nor blow up is known for the case $f(u)=|u|^{2}$ (except almost global existence, see [22]).

Let us give a short description of the idea of space-time resonances in the simple case $f(u, \bar{u})=u^{2}$, since $u$ is expected to have an asymptotically linear behavior, it is natural to consider $\tilde{u}=e^{-i t \Delta} u$, solution of

$$
\begin{aligned}
\tilde{u}(t) & =u_{0}-i \int_{0}^{t} e^{-i s \Delta}\left(e^{i s \Delta} \tilde{u}\right)\left(e^{i s \Delta} \tilde{u}\right) d s \\
\Leftrightarrow & \widehat{\widetilde{u}}(t)=\widehat{u_{0}}-i \int_{0}^{t} \int_{\mathbb{R}^{d}} e^{i s \Phi(\xi, \eta)} \widehat{\widetilde{u}}(s, \eta) \widehat{\vec{u}}(s, \xi-\eta) d \eta d s .
\end{aligned}
$$

with $\Phi(\xi, \eta)=|\xi|^{2}-|\eta|^{2}-|\xi-\eta|^{2}$. The existence of global solutions reduces to construct a fixed point to (A.2) in a suitable functional space, and thus estimate the integral in (A.3). As $\partial_{s} \tilde{u}=-i e^{-i s \Delta} u^{2}$, an integration by parts in the $s$ variable increases the nonlinearity in (A.3) and can be done if $\Phi$ does not vanish. Unfortunately, $\Phi$ vanishes on the large set $\{\eta \perp \xi-\eta\}$. Nevertheless, from the identity $e^{i s \Phi}=\frac{\nabla_{\eta} \Phi \cdot \nabla_{\eta} e^{i s \Phi}}{s\left|\nabla_{\eta} \Phi\right|^{2}}$, it is apparent that an integration by parts in the $\eta$ variable can be fruitful too as it adds the coefficient $\frac{1}{s}$, which improves the decay. This idea leads to the following definition:

Definition 1 The time resonant set is $\mathcal{T}=\{(\eta, \xi): \Phi(\eta, \xi)=0\}$.

The space resonant set is $\mathcal{S}=\left\{(\eta, \xi): \nabla_{\eta} \Phi(\eta, \xi)=0\right\}$.

The space-time resonant set is $\mathcal{R}=\mathcal{T} \cap \mathcal{S}$.

In our example, $\Phi$ vanishes iff $2 \eta \perp \xi$, while $\nabla_{\eta} \Phi$ vanishes if $\eta=\xi-\eta$. This implies for the space-time resonant set

$$
\mathcal{T} \cap \mathcal{S}=\{(\xi, \eta): \Phi(\xi, \eta)=0\} \cap\left\{(\xi, \eta): \nabla_{\eta} \Phi(\xi, \eta)=0\right\}=\{\xi=\eta=0\} .
$$

Thus using a convenient frequency partition, a gain by integration by parts can always be obtained. On the other hand this clarifies why it is hard to handle the nonlinearity $|u|^{2}$, indeed in this case $\Phi(\xi, \eta)=|\xi|^{2}+|\eta|^{2}-|\xi-\eta|^{2}$ and it is easily checked that $\mathcal{T} \cap \mathcal{S}=\{\xi=0\}$ which is of dimension 3 (in the phase space $(\eta, \xi) \in\left(\mathbb{R}^{3}\right)^{2}$ ). 
The concept of space-time resonances was introduced by Gustafson et al. [29] to obtain the scattering for the Gross-Pitaevskii equation. Independently Germain et al introduced it in [19] to give a new proof of scattering for (NLS) with $f(u, \bar{u})=u^{2}$ before tackling global existence for the gravity waves problem [20]. This strategy has had since numerous applications for the study of global well-posedness of dispersive equations (e.g., Euler-Poisson [26], capillary waves [21], or our recent work on the general Euler-Korteweg system [3]).

From elementary computation, we have the following proposition for the phase associated to the nonlinearity $|\varphi|^{2}$.

Proposition A.1 (spacetime resonances for (A.4)) The space resonant set for $\Phi(\eta, \xi)=$ $H(\xi)-H(\eta)+H(\xi-\eta)$ is $\{\xi=0\}$.

The spacetime resonant set for $\Phi(\eta, \xi)=H(\xi)-H(\eta)-H(\xi-\eta)$ is $\{\xi=0\}$.

The large size of the space-time resonant set is one of the reasons why scattering for (1.3) is a difficult issue.

\section{A.2 Normal form}

Let us rewrite the Gross-Pitaevskii equation for the variable $v=\varphi_{1}+i U \varphi_{2}$ :

$$
i \partial_{t} v-H v=U\left(3 \varphi_{1}^{2}+\varphi_{2}^{2}+|\varphi|^{2} \varphi_{1}\right)+i\left(2 \varphi_{1} \varphi_{2}+|\varphi|^{2} \varphi_{2}\right) .
$$

The $U$ in factor of the real part of the nonlinearity is a nice feature, indeed its symbol cancels at $\xi=0$, which is precisely the spacetime resonant set in the worst case (see Proposition A.1). Namely near $\xi=\varepsilon \eta, \eta<<1$ we have

$$
H(\varepsilon \eta)-H(\eta)+H((\varepsilon-1) \eta) \sim_{\epsilon \rightarrow 0,|\eta| \rightarrow 0} \frac{-3 \varepsilon|\eta|^{3}}{2 \sqrt{2}}=\frac{-3|\xi||\eta|^{2}}{2 \sqrt{2}} .
$$

In other words the $U$ in factor counterbalances the negative effects due to the space-time resonant set (indeed the nonlinearity $|\varphi|^{2}$ is present since for example $\varphi_{1}^{2}=\varphi^{2}+\bar{\varphi}^{2}+2|\varphi|^{2}$ ).

On the other hand we have the singular relation $\varphi_{2}=U^{-1} v_{2}$, and there is no good multiplier on the imaginary part which contains the quadratic, singular term $2 \varphi_{1} \varphi_{2}$. The normal form from Sect. 4.1 was the remedy to the singular relation, however the new nonlinearity has no good multiplier property, this is why Gustafson et al used the following different change of variable in [29]:

$$
Z=v-B_{1}^{\prime}\left[\varphi_{1}, \varphi_{1}\right]+B_{1}^{\prime}\left[\varphi_{2}, \varphi_{2}\right],
$$

where $B_{1}^{\prime}$ is the real valued symmetric bilinear Fourier multiplier of symbol $\frac{1}{2+\left|\xi_{1}\right|^{2}+\left|\xi_{2}\right|^{2}}$.

After some tedious computations one finds:

$$
i \partial_{t} Z-H Z=\mathcal{N}_{Z}(v),
$$

with

$$
\mathcal{N}_{Z}(v)=U\left(B_{1}\left[v_{1}, v_{1}\right]+B_{2}\left[v_{2}, v_{2}\right]\right)+C+Q,
$$

where $B_{1}, B_{2}$ are bilinear multipliers and $C$ (resp. $Q$ ) contains cubic (resp. quartic) terms. Thanks to this change of variables the new nonlinearity has both a "null structure" and smooth quadratic nonlinearities. Note however that cubic and quartic nonlinearities are not smooth (they are only smooth in $\varphi$ ). For later use, let us mention that the symbol of $B_{2}$ is

$$
B_{2}\left(\xi_{1}, \xi_{2}\right)=\frac{-2\left\langle\xi_{1}\right\rangle\left\langle\xi_{2}\right\rangle}{2+\left|\xi_{1}\right|^{2}+\left|\xi_{2}\right|^{2}} \frac{\xi_{1}}{\left|\xi_{1}\right|} \frac{\xi_{2}}{\left|\xi_{2}\right|}=B_{2}^{\prime}\left(\xi_{1}, \xi_{2}\right) \frac{\xi_{1}}{\left|\xi_{1}\right|} \frac{\xi_{2}}{\left|\xi_{2}\right|},
$$


that is the composition of the Riesz multipliers and a smooth bilinear multiplier $B_{2}^{\prime}$ (see Proposition 2.8).

\section{A.3 The functional space, reduction to a priori estimates}

Replacing in (A.3) $\Delta$ by $-H$, after an integration by parts in the $\eta$ variable, we get a factor $\nabla_{\eta}(\widetilde{u})=-i \mathcal{F}\left(x e^{i t H} u\right)$ which must be controlled. Furthermore, in view of the Strichartz estimates it is natural to use $\left\|U^{-1 / 6} v\right\|_{L_{t}^{2} W^{1,6}}$. Thus a natural functional space to study global well-posedness is:

$$
\begin{aligned}
\|Z\|_{X \cap S([0, T])} & =\sup _{0 \leq t \leq T}\|Z(t)\|_{X(t)}+\|Z\|_{S([0, T])} \\
& =\sup _{0 \leq t \leq T}\left(\|Z(t)\|_{H^{1}}+\left\|e^{-i t H} x e^{i t H} Z\right\|_{H^{1}}\right)+\left\|U^{-1 / 6} Z\right\|_{L_{T}^{2} W^{1,6}} .
\end{aligned}
$$

For $\psi(0) \in 1+H^{1}$, global well-posedness is well-known (e.g., see the appendix of [8]), thus the main goal is to prove a priori estimates for the map

$$
Z \mapsto e^{-i t H} Z(0)-i \int_{0}^{t} e^{-i(t-s) H} \mathcal{N}_{Z}(v)(s) d s .
$$

More precisely it suffices that for some $k \geq 0$

$$
\begin{aligned}
& \left\|e^{-i t H} Z(0)-i \int_{0}^{t} e^{-i(t-s) H} \mathcal{N}_{Z}(v)(s) d s\right\|_{X \cap S([0, T])} \lesssim\left\|Z_{0}\right\|_{X(0)} \\
& \quad+\|Z\|_{X \cap S([0, T])}^{2}\left(1+\|Z\|_{X \cap S([0, T])}^{k}\right) .
\end{aligned}
$$

with constants independent of $T$ so that a standard bootstrap argument implies global wellposedness and scattering ${ }^{4}$. In the rest of this appendix we shortly summarize the strategy: bounding $\|Z\|_{S}$ is relatively elementary and is explained in Sect. A.4. The main issue is the control of $x e^{i t H} Z$, and more precisely the so-called bilinear terms with phase derivative from [29]. The main ideas (estimates on singular bilinear multipliers and the decomposition of the phase space $(\eta, \xi)$ in "non-resonant" regions) are described in Sect. A.5.

\section{A.4 Control of the $S$ norm, action of the normal form}

This section summarizes the estimates that can be obtained simply by using only standard product rules, functional spaces embeddings, Strichartz estimates and dispersive estimates of the form (2.15) and (2.16). They cover two points: the normal form is a well-defined change of variables for small data, and the $S$ norm of the map (A.7) is controlled by the $S \cap X$ norm of $Z$. First let us give a list of quantities controlled by the $X \cap S$ norm.

Proposition A.2 Let $v_{<1}=\mathcal{F}^{-1}\left(1_{\{|\xi|<1\}} \widehat{v}(\xi)\right)$ and $v_{\geq 1}=\mathcal{F}^{-1}\left(1_{\{|\xi| \geq 1\}} \widehat{v}(\xi)\right)$. For $0 \leq \theta \leq$ 1 , we have the following estimates

$$
\|v(t)\|_{\dot{H}^{-1}} \lesssim\|v(t)\|_{X(t)},
$$

4 Actually in [27] the authors obtain some time decay:

$$
\left\|Z-e^{-i\left(t-T_{0}\right) H} Z\left(T_{0}\right)\right\|_{X \cap S\left(T_{0},+\infty\right)} \lesssim\left\langle T_{0}\right\rangle^{-\varepsilon}\|Z\|_{X \cap S\left(T_{0}, \infty\right)}^{2}\left(1+\|Z\|_{X \cap S\left(T_{0}, \infty\right)}\right) .
$$




$$
\begin{aligned}
& \left\|U^{-2} v\right\|_{L^{6}} \lesssim\|v(t)\|_{X(t)} \lesssim\|v(t)\|_{X(t)}, \\
& \left\||\nabla|^{-2+\frac{5 \theta}{3}} v_{<1}(t)\right\|_{L^{6}} \lesssim \min \left(1, t^{-\theta}\right)\|v(t)\|_{X(t)}, \\
& \left\||\nabla|^{\theta} v_{\geq 1}(t)\right\|_{L^{6}} \lesssim \min \left(t^{-\theta}, t^{-1}\right)\|v(t)\|_{X(t)} .
\end{aligned}
$$

and $U^{-1} v$ satisfies dispersion/Strichartz type estimates

$$
\begin{aligned}
& \left\|U^{-1} v(t)\right\|_{L^{6}} \lesssim\langle t\rangle^{-\frac{3}{5}}\|v(t)\|_{X(t)}, \\
& \left\|U^{-1} v\right\|_{L_{t}^{2}\left(H^{1,6}\right)} \lesssim\|v\|_{X(t) \cap S(t)}, \\
& \left\|\langle\nabla\rangle^{\frac{2}{3}} U^{-1} v(t)\right\|_{L^{4}} \lesssim t^{-\frac{7}{12}}\|v(t)\|_{X(t)} .
\end{aligned}
$$

We have also control of Strichartz norms of $\varphi$ in terms of the norm of $\|v\|_{X \cap S}$.

Proposition A.3 We have the following estimates:

$$
\begin{aligned}
\|\varphi\|_{L^{\infty}\left(H^{1}\right)} & \lesssim\|v\|_{X}, \\
\|\varphi\|_{L^{2}\left(H^{1,6}\right)} & \lesssim\|v\|_{X \cap S .}
\end{aligned}
$$

Estimates on the normal form Using the estimates above and basic product rules, one can prove that the normal form $v \mapsto Z$ is a well-defined change of variables near 0 in our functional settings, and that the smallness condition on $\varphi_{0}$ is equivalent to the smallness in $X(0)$ of $Z_{0}$ (we refer to (1.14) for this condition). The fact that the map $v \rightarrow Z$ is bi-Lipschitz in $X_{\infty} \cap S_{\infty}$ is crucial since we need to estimate the nonlinearity $\mathcal{N}_{Z}(v)$ (which depends a priori only on $v$ ) in norms of $Z$ in $X_{\infty} \cap S_{\infty}$.

Proposition A.4 ([29] Sects. 6, 7). There exists $\varepsilon>0$ such uniformly in $T>0$, the map

$$
\begin{aligned}
& X \cap S([0, T]) \mapsto X \cap S([0, T]), \\
& v \mapsto Z=v+B_{1}^{\prime}\left[\varphi_{1}, \varphi_{1}\right]+B_{1}^{\prime}\left[\varphi_{2}, \varphi_{2}\right]=v+b(\varphi),
\end{aligned}
$$

is bi-Lipschitz from $B(0, \varepsilon)$ to its image, in particular

$$
\begin{aligned}
& X(0) \mapsto X(0), \\
& v_{0} \mapsto v_{0}+b\left(\varphi_{0}\right),
\end{aligned}
$$

is bi-Lipschitz $B(0, \varepsilon)$ to its image, and under the same smallness condition $v$ and $Z$ have the same asymptotics:

$$
\|v-Z\|_{S \cap X\left(\left[T_{1}, T_{2}\right]\right.} \lesssim T_{1}^{-1 / 6}\|Z\|_{S \cap X\left(\left[T_{1}, T_{2}\right]\right)} .
$$

For $\left\|Z_{0}\right\|_{X(0)}$ small enough we have $\left\|Z_{0}\right\|_{X(0)} \sim\left\|\langle x\rangle \nabla \varphi_{0}\right\|_{L^{2}}+\left\|\langle x\rangle \operatorname{Re}\left(\varphi_{0}\right)\right\|_{L^{2}}$.

\section{Control of the $S$ norm}

Proposition A.5 For all $T_{2} \geq T_{1} \geq 0$ we have:

$$
\left\|\int_{T}^{t} e^{-i(t-s) H} \mathcal{N}_{Z}(v) d s\right\|_{S\left(\left[T_{1}, T_{2}\right]\right)} \lesssim\left\langle T_{1}\right\rangle^{-1 / 2}\left(\|v\|_{X \cap S\left(\left[T_{1}, T_{2}\right]\right)}^{2}+\|v\|_{X \cap S\left(\left[T_{1}, T_{2}\right]\right)}^{4}\right),
$$


Sketch of proof We only show how to bound $\int_{T_{1}}^{t} e^{-i(t-s) H} B_{2}\left[v_{2}, v_{2}\right] d s$ for $T_{1} \geq 1$ (for finite time it suffices to use the results of strong solution in finite time). The Strichartz estimate of Proposition 2.17 gives:

$$
\begin{aligned}
& \left\|U^{\frac{-1}{6}} \int_{T}^{t} e^{-i(t-s) H} B_{2}\left[v_{2}, v_{2}\right] d s\right\|_{L^{2}\left(\left[T_{1}, T_{2}\right] H^{1,6}\right)} \lesssim\left\|B_{2}\left[v_{2}, v_{2}\right]\right\|_{L^{1}\left(\left[T_{1}, T_{2}\right], H^{1}\right)}, \\
& \left\|\int_{T}^{t} e^{-i(t-s) H} B_{2}\left[v_{2}, v_{2}\right] d s\right\|_{L_{t>T}^{\infty}\left(H^{1}\right)} \lesssim\left\|B_{2}\left[v_{2}, v_{2}\right]\right\|_{L_{t>T}^{1}\left(H^{1}\right)} .
\end{aligned}
$$

Let $R_{1}, R_{2}$ Riesz operator, using Proposition 2.8, Hölder's inequality, interpolation and $\|v(t)\|_{L^{6}} \lesssim t^{-1}\|v\|_{X \cap S\left(\left[T_{1}, T_{2}\right]\right)}($ see (A.12)) leads to

$$
\begin{aligned}
\left\|B_{2}\left[v_{2}, v_{2}\right]\right\|_{L^{1}\left(\left[T_{1}, T_{2}\right], H^{1}\right)} & \lesssim\left\|R_{1} v_{2}\right\|_{L^{4}\left(\left[T_{1}, T_{2}\right], W^{1,3}\right)}\left\|R_{2} v_{2}\right\|_{L^{4 / 3}\left(\left[T_{1}, T_{2}\right], L^{6}\right)} \\
& \lesssim T_{1}^{-1 / 2}\|v\|_{X \cap S\left(\left[T_{1}, T_{2}\right]\right)}^{2} .
\end{aligned}
$$

\section{A.5 Control of the $X$ norm}

In order to estimate the $X([0, T])$ norm of (A.7), the main issue is to control

$$
\left\|x e^{i t H} \int_{0}^{t} e^{-i(t-s) H} \mathcal{N}_{Z}(v)(s) d s\right\|_{H^{1}}=\left\|\nabla_{\xi}(D) \int_{0}^{t} e^{i s H(\xi)} \mathcal{F}\left(\mathcal{N}_{Z}(v)(s)\right) d s\right\|_{H^{1}} .
$$

For simplicity, we focus on the quadratic term $B_{2}\left(Z_{2}, Z_{2}\right)$. Following [29], for any complex we denote $z^{+}=z, z^{-}=\bar{z}$. Since $Z_{2}=\left(Z^{+}-Z^{-}\right) /(2 i)$, the generic term to estimate is $B_{2}\left[Z^{ \pm}, Z^{ \pm}\right]$. Let us denote the Fourier transform of the profile $\widetilde{Z^{ \pm}}=e^{ \pm i t H(\xi)} \mathcal{F}\left(Z^{ \pm}\right)(\xi)$, we have to control in particular the following term:

$$
\int_{0}^{t} \int_{\mathbb{R}^{d}} \nabla_{\xi}\left(e^{i s(H(\xi) \mp H(\eta) \mp H(\xi-\eta)} B_{2}(\eta, \xi-\eta) \widetilde{Z^{ \pm}}(\eta) \widetilde{Z}^{ \pm}(\xi-\eta)\right) d \eta d s .
$$

We set $\Omega_{ \pm \pm}(\eta, \xi)=H(\xi) \mp H(\eta) \mp H(\xi-\eta)$. As was pointed out in Sect. A.1, the worst case in term of spacetime resonances is the +- case, where the resonant set is $\{\xi=0\}$. Finally, let us point out that when $\nabla_{\xi}$ falls on $e^{i s H(\xi) \pm H(\eta) \pm H(\xi-\eta)}$, we get the term:

$$
\left.\int_{0}^{t} \int_{\mathbb{R}^{d}} i s \nabla_{\xi} \Omega_{ \pm \pm} e^{i s \Omega_{ \pm \pm}} B_{2}(\eta, \xi-\eta) \widetilde{Z^{ \pm}}(\eta) \widetilde{Z}^{ \pm}(\xi-\eta)\right) d \eta d s
$$

This is the worst as we both have a loss of decay due to the $s$ factor and a loss of derivative (in high frequencies) due to $\nabla_{\xi} \Omega$, and indeed it is easily checked that for this term a direct approach only relying on Strichartz estimate and the decay provided by the $X$ norm fails. This is the point where integration by parts in $\eta$ or $s$ is required. The last two paragraphs focus on the two ideas developed in [29] to effectively do it:

- partition of the phase space in time non-resonant and space non-resonant areas: there is a decomposition of the symbol $B_{2}=B_{2}^{X}+B_{2}^{T}$ such that (in some sense precised in Lemma A.7) $B_{2}^{X}$, resp. $B_{2}^{T}$, is supported in a non-space, resp. time, resonant area. Furthermore it is convenient to localize in frequency by introducing for $j \in \mathbb{Z}$ LittlewoodPaley functions $\chi_{j}$, and $B_{2}^{a, b, c, T}=\chi_{a}(\xi) \chi_{b}(\eta) \chi_{c}(\xi-\eta) B_{2}(\eta, \xi-\eta)$, and similarly $B_{2}^{a, b, c, T}$. 
- Once the integration by parts are performed, the new terms obtained are estimated thanks to a bilinear multiplier estimate with losses for non-smooth multipliers. We also mention why this estimate cannot be avoided.

The bilinear multiplier estimate For conciseness we write $\Omega$ instead of $\Omega_{ \pm \pm}$. Using the partition of unity $1=\sum \chi_{a}(\xi) \chi_{b}(\eta) \chi_{c}(\xi-\eta)$, one is reduced to estimate each integral:

$$
\left.I_{a b c}=\int_{0}^{t} \int_{\mathbb{R}^{d}} i s \nabla_{\xi} \Omega_{ \pm \pm} e^{i s \Omega_{ \pm \pm}} B_{2}^{a, b, c}(\eta, \xi-\eta) \widetilde{Z^{ \pm}}(\eta) \widetilde{Z}^{ \pm}(\xi-\eta)\right) d \eta d s .
$$

An integration by parts in $\eta$ or $s$ can be done thanks to the identities:

$$
e^{i s \Omega}=\frac{\nabla_{\eta} \Omega}{i s\left|\nabla_{\eta} \Omega\right|^{2}} \cdot \nabla_{\eta} e^{i s \Omega}, \quad e^{i s \Omega}=\frac{1}{i \Omega} \partial_{s} e^{i s \Omega} .
$$

To be fruitful, of course it is necessary that $\nabla_{\eta} \Omega_{ \pm \pm}$, resp. $\Omega$, does not vanish on $\operatorname{supp}\left(B^{a, b, c, X}\right), \operatorname{resp} . \operatorname{supp}\left(B^{a, b, c, T}\right)$, but this is not sufficient. In order to apply the celebrated Coifman-Meyer theorem, one needs to have $\left|\partial^{\alpha} B\right| \lesssim(|\eta|+|\xi|)^{-|\alpha|}$. Such an estimate is hopeless as can be seen from an elementary computation: near $\xi=\varepsilon \eta$ with $\varepsilon,|\eta|<<1$, we have

$$
\begin{aligned}
& H(\varepsilon \eta)-H(\eta)+H((\varepsilon-1) \eta) \sim \frac{-3 \varepsilon|\eta|^{3}}{2 \sqrt{2}}=\frac{-3|\xi||\eta|^{2}}{2 \sqrt{2}}, \\
& \text { while }|\varepsilon \eta|^{2}-|\eta|^{2}+|(1-\varepsilon) \eta|^{2} \sim-2|\eta||\xi|
\end{aligned}
$$

thus there is no hope for $1 / \Omega$ to belong to any standard class of multipliers.

The remedy from [29] is the following multiplier estimate which only requires a very weak regularity for the multiplier:

Lemma A.6 Let $0 \leq s \leq \frac{N}{2},(p, q)$ any dual-Strichartz exponent except for the endpoint namely

$$
\frac{2}{p}+\frac{N}{q}=2+\frac{N}{2}, p<2
$$

Let $\left(p_{1} q_{1}\right)$ and $\left(p_{2}, q_{2}\right)$ satisfy:

$$
\begin{aligned}
& \frac{1}{p}=\frac{1}{p_{1}}+\frac{1}{p_{2}}, \frac{1}{q}+\frac{1}{q(s)}=\frac{1}{q_{1}}+\frac{1}{q_{2}}, \frac{1}{q(s)}=\frac{1}{2}-\frac{s}{N}, \\
& p \leq p_{1}, p_{2} \leq+\infty, q \leq q_{1}, q_{2} \leq+\infty
\end{aligned}
$$

Then for any bilinear Fourier multiplier we have:

$$
\left\|\int e^{i t H} B[u, v] d t\right\|_{L^{2}} \lesssim\|B\|_{\tilde{L}_{\xi}^{\infty} \dot{B}_{2,1, \eta}^{s}}+\widetilde{L}_{\xi}^{\infty} \dot{B}_{2,1, \zeta}^{s}\|u\|_{L^{p_{1}} L^{q_{1}}}\|v\|_{L^{p_{2}} L^{q_{2}}} .
$$

with the first norm of $B$ is in the $(\xi, \eta)$ coordinates and the second in the $(\xi, \zeta)=(\xi, \xi-\eta)$.

If $1 / q_{1}+1 / q_{2}=1 / 2+1 / q(s), 2 \leq q_{1}, q_{2} \leq q(s), \frac{1}{q(s)}=\frac{1}{2}-\frac{s}{N}$, then for any bilinear Fourier multiplier:

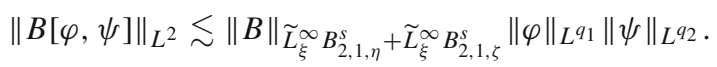

We recall here the definition of Chemin-Lerner spaces:

$$
\|f(\xi, \eta)\|_{\widetilde{L}_{\xi}^{p}\left(\dot{B}_{q, r, \eta}^{s}\right)}=\left(\sum_{l \in \mathbb{Z}} 2^{l s r}\|f\|_{L_{\xi}^{p}\left(L_{\eta}^{q}\right)}^{r}\right)^{\frac{1}{r}} .
$$


Remark 16 When $s=\frac{N}{2}$ Lemma A.6 is similar to a classical Strichartz estimates where $B[u, v]$ behaves like a classical product $u v$ (in high frequencies it is in particular sufficient to work with $s=\frac{N}{2}$ since the classical Strichartz estimates are sufficient to estimate the quadratic nonlinearities). Let us observe that $q(s)$ is the Sobolev exponent for the embedding $\dot{B}_{2,1}^{s} \subset L^{q(s)}$ and $\frac{1}{q(s)}$ gives the precise loss compared with Hölder inequality, in particular it implies that when $0 \leq s<\frac{N}{2}$ we need a better long-time decay to use this result compared with the classical Strichartz estimate.

Remark 17 It is actually simpler to control the $\widetilde{L}^{\infty}\left(H^{s}\right)$ norm by the $L^{\infty}\left(H^{s+\epsilon}\right)$ norm with $\epsilon>0$.

We set $\left[B^{s}\right]=\widetilde{L}_{\xi}^{\infty} B_{2,1, \eta}^{s}+\widetilde{L}_{\xi}^{\infty} B_{2,1, \zeta}^{s}$, with the lemma in hand, it is now a matter of checking that for any $(a, b, c)$ either

$$
\left.\left\|\frac{\nabla_{\eta} \Omega \cdot \nabla_{\xi} \Omega}{\left|\nabla_{\eta} \Omega\right|^{2}} B_{2}^{a, b, c, X}\right\|_{\left[B^{s}\right]}, \| \nabla_{\eta}\left(\frac{\nabla_{\eta} \Omega \cdot \nabla_{\xi} \Omega}{\left|\nabla_{\eta} \Omega\right|^{2}} B_{2}^{a, b, c, X}\right)\right) \|_{\left[B^{s}\right]} \text { or }\left\|\frac{\nabla_{\xi} \Omega}{\Omega} B_{2}^{a, b, c, T}\right\|_{\left[B^{s}\right]},
$$

satisfies "good" estimates. 5

The phase space partition and consequence. Following (A.20), we define

$$
\begin{gathered}
\mathcal{B}_{1,2}^{a, b, c, X}=\frac{\nabla_{\eta} \Omega \cdot \nabla_{\xi} \Omega}{\left|\nabla_{\eta} \Omega\right|^{2}} B_{2}^{a, b, c, X}, \mathcal{B}_{2,2}^{a, b, c, X}=\nabla_{\eta}\left(\frac{\nabla_{\eta} \Omega \cdot \nabla_{\xi} \Omega}{\left|\nabla_{\eta} \Omega\right|^{2}} B_{2}^{a, b, c, X}\right), \\
\mathcal{B}_{3,2}^{a, b, c, T}=\frac{\nabla_{\xi} \Omega}{\Omega} B_{2}^{a, b, c, T} .
\end{gathered}
$$

The crucial result is the following Lemma (which is also true for the quadratic nonlinearity $\left.\left.U B_{1}\left[v_{1}, v_{1}\right]\right)\right)$.

Lemma A.7 Let $M=\max (a, b, c), m=\min (a, b, c)$ and $l=\min (b, c)$. Then

- For any $(a, b, c) \in \mathbb{Z}^{3}$

$$
\begin{aligned}
& \text { for } M<<1,0<\varepsilon<<1,\left\|\mathcal{B}_{1,2}^{a, b, c, X}\right\|_{H^{1+\epsilon}} \lesssim l^{\frac{1}{2}-2 \epsilon}, \quad\left\|\mathcal{B}_{2,2}^{a, b, c, X}\right\|_{H^{1+\epsilon}} \lesssim l^{\frac{1}{2}-2 \epsilon} M^{-1}, \\
& \text { for } M \geq 1,|\varepsilon|<<1,\left\|\mathcal{B}_{1,2}^{a, b, c, X}\right\|_{H^{\frac{3}{2}+\epsilon}} \lesssim l^{1-\epsilon}\langle a\rangle^{-1}, \quad\left\|\mathcal{B}_{2,2}^{a, b, c, X}\right\|_{H^{\frac{3}{2}+\epsilon}} \lesssim l^{-\epsilon}\langle a\rangle^{-1},
\end{aligned}
$$

- For any $(a, b, c) \in \mathbb{Z}^{3}, 0<s<2$,

$$
\left\|\mathcal{B}_{3,2}^{a, b, c, T}\right\|_{\left[B^{s}\right]} \lesssim\left(\frac{\langle M\rangle}{M}\right)^{s} l^{\frac{3}{2}-s}\langle a\rangle^{-1} .
$$

For the sake of completeness, let us illustrate how this lemma can be used to control a typical non-time resonant term. By integrations by parts in $s$ we observe that we have to estimate terms of the form:

$$
\begin{aligned}
& \int_{0}^{T} e^{i s H} U\left(\mathcal{B}_{3,2}^{a, b, c, T}\left[Z^{ \pm}, s \mathcal{N}_{Z}^{ \pm}\right]+\mathcal{B}_{3,2}^{a, b, c, T}\left[s \mathcal{N}_{Z}^{ \pm}, Z^{ \pm}\right]\right) d s \text { or } \\
& \int_{0}^{T} e^{i s H} U \mathcal{B}^{a, b, c, T}\left[Z^{ \pm}, Z^{ \pm}\right] d s
\end{aligned}
$$

5 "Good" means that combining these estimates with Lemma A.6 is sufficient to close the estimates on (A.7). 
Applying Lemma A.6, Bernstein's lemma and using the fact that $U$ is increasing we have:

$$
\begin{aligned}
& \left\|\int_{0}^{T} e^{i s H}\left(\sum_{b \lesssim a \sim c} \mathcal{B}_{3}^{a, b, c}[s \mathcal{N} Z, Z]+\mathcal{B}_{3}^{a, b, c}[Z, s \mathcal{N} Z]\right) d s\right\|_{H^{1}} \\
& \lesssim \sum_{b \lesssim a \sim c} U(b)^{\frac{1}{2}} U(c)\langle a\rangle\langle b\rangle^{-1}\langle c\rangle^{-1}\left\|\mathcal{B}_{3}^{a, b, a}\right\|_{\left[B^{\frac{1}{2}+\epsilon}\right]+\left[B^{3 / 2}\right]}\left\|U^{-\frac{1}{2}}\langle\nabla\rangle t \mathcal{N}_{Z}\right\|\left(L_{T}^{\frac{1}{1-\epsilon / 2}} \cap L_{T}^{2}\right)\left(L^{3}\right) \\
& \times\left\|U^{-1}\langle\nabla\rangle Z\right\|_{L_{T}^{\infty}\left(L^{2}\right)} \\
& \lesssim \sum_{b \lesssim a \sim c} U(b)^{\frac{1}{2}} U(a)\langle b\rangle^{-1}\left\|\mathcal{B}_{3}^{a, b, a}\right\|\left[B^{\frac{1}{2}+\epsilon}\right]+\left[B^{3 / 2}\right]^{\left\|U^{-\frac{1}{2}}\langle\nabla\rangle t \mathcal{N}_{Z}\right\|}\left(L_{T}^{\frac{1}{1-\epsilon / 2}} \cap L_{T}^{2}\right)\left(L^{3}\right) \\
& \times\left\|U^{-1}\langle\nabla\rangle Z\right\|_{L_{T}^{\infty}\left(L^{2}\right)} .
\end{aligned}
$$

Let us deal now with the case $a<<1$ which gives:

$$
\begin{aligned}
& \sum_{b \lesssim a<<1} U(b)^{\frac{1}{2}} U(a)\langle b\rangle^{-1}\left\|\mathcal{B}_{3}^{a, b, a}\right\|_{\left[H^{1+\epsilon}\right]} \\
& \lesssim \sum_{a<<1} \sum_{b \lesssim a} U(b)^{\frac{1}{2}} U(a)\langle b\rangle^{-1}\left(\frac{\langle a\rangle}{a}\right)^{1+\epsilon} b^{\frac{1}{2}-\epsilon}\langle a\rangle^{-1} \\
& \lesssim \sum_{a<<1} \sum_{b \lesssim a} b^{\frac{1}{2}} a a^{-1-\epsilon} b^{\frac{1}{2}-\epsilon} \lesssim \sum_{a<<1} a^{-\epsilon} \sum_{b \lesssim a} b^{1-\epsilon} \lesssim \sum_{a<<1} a^{-\epsilon} a^{1-\epsilon} \lesssim 1
\end{aligned}
$$

and for $a \geq 1$ we get with $|\epsilon|>0$ small enough:

$$
\begin{aligned}
& \sum_{b \lesssim a, a \gtrsim 1} U(b)^{\frac{1}{2}} U(a)\langle b\rangle^{-1}\left\|\mathcal{B}_{3}^{a, b, a}\right\|\left[H^{\frac{3}{2}+\epsilon}\right] \\
& \lesssim \sum_{a \gtrsim 1} \sum_{b \lesssim a} U(b)^{\frac{1}{2}} U(a)\langle b\rangle^{-1}\left(\frac{\langle a\rangle}{a}\right)^{\frac{3}{2}+\epsilon} b^{-\epsilon}\langle a\rangle^{-1}, \\
& \lesssim \sum_{a \gtrsim 1}\langle a\rangle^{-1} \sum_{b \lesssim a} U(b)^{\frac{1}{2}}\langle b\rangle^{-1} b^{-\epsilon} \lesssim 1 .
\end{aligned}
$$

On the other hand (A.9),(A.11) give

$$
\begin{aligned}
\left\|U^{-1} Z\right\|_{L^{\infty} L^{2}} & \lesssim\|Z\|_{X([0, \infty[)}, \\
\left\|U^{1 / 2} t Z^{2}\right\|_{L^{1-\epsilon / 2} L^{3}} & \lesssim\|t\| Z\left\|_{L^{6}}^{2}\right\|_{L^{\frac{1}{1-\varepsilon / 2}}} \lesssim\left\|t^{-1}\right\|_{L^{\frac{1}{1-\varepsilon / 2}}}\|Z\|_{X([0, \infty[}^{2} \lesssim\|Z\|_{X([0, \infty[}^{2} .
\end{aligned}
$$

To finish let us describe briefly the delicate case when $a \leq b \sim c$ which corresponds to the space-time resonant set. Let us consider in particular the most difficult term $\sum_{a \lesssim b \sim c} \int_{0}^{t} e^{i s H}\left(\mathcal{B}_{3,2}^{a, b, c}\left[Z^{+}, Z^{-}\right]\right) d s$, applying again Lemma A.6, Bernstein's lemma and the fact that $B_{2,2}^{\epsilon}$ is embedded in $B_{2,1}^{0}$ for non-homogeneous Besov space, we have:: 


$$
\begin{aligned}
& \left\|\sum_{a \lesssim b \sim c} \int_{0}^{t} e^{i s H}\left(\mathcal{B}_{3,2}^{a, b, c}\left[Z^{+}, Z^{-}\right]\right) d s\right\|_{H^{1}} \lesssim \sum_{b} \sum_{a \lesssim b}\left\|\int_{0}^{t} e^{i s H}\left(\langle a\rangle \mathcal{B}_{3,2}^{a, b, b}\left[Z^{+}, Z^{-}\right]\right) d s\right\|_{L^{2}} \\
& \lesssim \sum_{b}\left\|\int_{0}^{t} e^{i s H} \sum_{a \lesssim b}\left(\langle a\rangle \mathcal{B}_{3,2}^{a, b, b}\left[Z^{+}, Z^{-}\right]\right) d s\right\|_{H^{\epsilon}} \\
& \lesssim \sum_{b}\langle b\rangle^{\epsilon^{\prime}}\left\|\int_{0}^{t} e^{i s H} \sum_{a \lesssim b}\left(\langle a\rangle \mathcal{B}_{3,2}^{a, b, b}\left[Z^{+}, Z^{-}\right]\right) d s\right\|_{L^{2}} \\
& \lesssim \sum_{b<<1} U(b) U(b)^{\frac{1}{6}} \\
& \langle b\rangle^{-2}\left\|\sum_{a \lesssim b}\langle a\rangle \mathcal{B}_{3,2}^{a, b, b}\right\|_{\left[B^{1+\epsilon}\right]}\left\|\langle\nabla\rangle U^{-1} Z^{+}\right\|_{L^{\infty}\left(L^{2}\right)}\left\|\langle\nabla\rangle U^{-1 / 6} Z^{-}\right\| \frac{1}{L^{\frac{1}{1-\frac{\epsilon}{2}}\left(L^{6}\right)}} \\
& +\sum_{b \gtrsim 1} U(b) U(b)^{\frac{1}{6}}\langle b\rangle^{-2+\epsilon^{\prime}}\left\|\sum_{a \lesssim b}\langle a\rangle \mathcal{B}_{3,2}^{a, b, b}\right\|_{\left[B^{\frac{3}{2}}\right]} \| \\
& \langle\nabla\rangle U^{-1} Z^{+}\left\|_{L^{\infty}\left(L^{2}\right)}\right\|\langle\nabla\rangle U^{-1 / 6} Z \|_{L^{4 / 3}\left(L^{6}\right)} .
\end{aligned}
$$

Let us deal now with the case $b<<1$, we have then:

$$
\begin{aligned}
& \sum_{b<<1} U(b) U(b)^{\frac{1}{6}}\langle b\rangle^{-2}\left\|\sum_{a \lesssim b}\langle a\rangle \mathcal{B}_{1, j}^{a, b, b}\right\|_{\left[H^{1+\epsilon}\right]} \lesssim \sum_{b<<1} U(b) U(b)^{\frac{1}{6}} \sup _{a \lesssim b}\left\|\langle a\rangle \mathcal{B}_{1, j}^{a, b, b}\right\|_{\left[H^{1+\epsilon}\right]}, \\
& \lesssim \sum_{b<<1} U(b) U(b)^{\frac{1}{6}}\left(\frac{\langle b\rangle}{b}\right)^{1+\epsilon} b^{\frac{1}{2}-\epsilon} \lesssim \sum_{b<<1} b^{\frac{7}{6}} b^{-\frac{1}{2}-2 \epsilon} \lesssim 1
\end{aligned}
$$

We obtain similar estimates for the case $b \gtrsim 1$.

Conclusion. Collecting bounds similar to the one above eventually leads to (A.8), the same argument works if we replace $[0, \infty[$ by $[0, T]$, so that if the initial data are small enough, $Z$ must remain small in $X \cap S([0, T]$ uniformly in $T>0$. In particular (see (A.15)) the solution scatters.

\section{Appendix B: Some remarks on the sharpness}

As scattering for the Gross-Pitaevskii equation holds for small data, there are two natural questions: what is the optimal functional space $X_{0}$ for the initial data, and in such settings is there an optimal constant such $\left\|\psi_{0}\right\|_{X_{0}}<c$ implies scattering of the solution? In the present state of the art, those questions seem out of reach, but we mention here what the existing theory allows to guess.

First, we point out that all the traveling wave with finite energy have (1.14) finite. Indeed let us recall that in their work Jones et al. [32,33] claim that the traveling wave have the following asymptotic expansion in space (see [25] for a rigorous proof):

$$
\psi_{c}\left(x_{1}, x_{\perp}\right) \sim 1+\frac{i \alpha x_{1}}{\left(x_{1}^{2}+\left(1-\frac{c^{2}}{2}\right)\left|x_{\perp}\right|^{2}\right)^{\frac{N}{2}}}+o\left(1 /|x|^{N-1}\right) .
$$

In particular $\nabla \psi_{c}$ is $O\left(\frac{1}{|x|^{N}}\right)$ so that $\langle x\rangle \nabla \psi_{c} \in L^{2}$ for $N \geq 3$. 
The smallness condition in Theorem 1.1 and 1.2 is not precisely quantified. Since the energy of traveling waves has a lower bound for $N \geq 3$ (see [7]), and as they might be the "minimal nonlinear objects", the following conjecture was made in [29]:

Conjecture Let $N=3$. For any global solution $\psi \in C\left(\mathbb{R}, 1+E_{1}\right)$ of $(G P)$ satisfying $E(\psi)<\mathcal{E}_{0}$ there is a unique $z_{+} \in H^{1}\left(\mathbb{R}^{3}\right)$ satisfying $E(\psi)=\left\|\langle\nabla\rangle z_{+}\right\|_{L^{2}}^{2}$ and

$$
\left\|M(\varphi(t))-e^{-i t H} z_{+}\right\|_{H^{1}\left(\mathbb{R}^{3}\right)} \rightarrow_{t \rightarrow+\infty} 0,
$$

with $M(\varphi)=v+\langle\nabla\rangle^{-2}|\varphi|^{2}$. Moreover, the map $\varphi(0) \rightarrow z_{+}$is a homeomorphism between the open balls of radius $\mathcal{E}_{0}^{\frac{1}{2}}$ around 0 in $E_{1}$ and $H^{1}$.

Remark 18 Let us recall that setting $\psi^{\kappa_{1}}$ :

$$
\psi^{\kappa_{1}}(t, x)=\psi\left(\frac{t}{\kappa_{1}}, \frac{\sqrt{2} x}{\kappa_{1}}\right),
$$

where $\psi$ is the solution of Theorem 1.2 then if $\psi$ is solution of $(1.3), \psi^{\kappa_{1}}$ is solution of the following Gross-Pitaevski equation:

$$
i \kappa_{1} \partial_{t} \psi^{\kappa_{1}}+\frac{\kappa_{1}^{2}}{2} \Delta \psi^{\kappa_{1}}=\left(\left|\psi^{\kappa_{1}}\right|^{2}-1\right) \psi^{\kappa_{1}}
$$

In particular the smallness Assumption (1.14) corresponds to:

$$
\int_{\mathbb{R}^{3}}\left\langle\sqrt{2} x / \kappa_{1}\right\rangle^{2}\left(\left|\operatorname{Re}\left(\varphi_{0}^{\kappa_{1}}\right)\right|^{2}+\frac{\kappa_{1}^{2}}{2}\left|\nabla \varphi_{0}^{\kappa_{1}}\right|^{2}\right)<\left(\frac{\kappa_{1}}{\sqrt{2}}\right)^{3} \delta,
$$

By using the Madelung transform $\psi^{\kappa_{1}}(t, x)=\sqrt{\rho}(t, x) e^{i \frac{\varphi(t, x)}{\kappa_{1}}}$, then $(\rho, \nabla \varphi)$ is solution of system (1.1) with $\kappa_{1}$. When $\kappa_{1}$ decreases, the condition becomes more and more restrictive.

\section{References}

1. Antonelli, P., Marcati, P.: On the finite energy weak solutions to a system in quantum fluid dynamics. Commun. Math. Phys. 287(2), 657-686 (2009)

2. Antonelli, P., Marcati, P.: The quantum hydrodynamics system in two space dimensions. Arch. Ration. Mech. Anal. 203(2), 499-527 (2012)

3. Audiard, C., Haspot, B.: Global well-posedness of the Euler-Korteweg system for small irrotational data. Commun. Math. Phys. 351(1), 201-247 (2017)

4. Bahouri, H., Chemin, J.-Y., Danchin, R.: Fourier Analysis and Nonlinear Partial Differential Equations, Volume 343 of Grundlehren der Mathematischen Wissenschaften [Fundamental Principles of Mathematical Sciences]. Springer, Heidelberg (2011)

5. Benzoni-Gavage, S., Danchin, R., Descombes, S.: On the well-posedness for the euler-korteweg model in several space dimensions. Indiana Univ. Math. J. 56, 1499-1579 (2007)

6. Béthuel, F., Danchin, R., Smets, D.: On the linear wave regime of the Gross-Pitaevskii equation. J. Anal. Math. 110, 297-338 (2010)

7. Béthuel, F., Gravejat, P., Saut, J.-C.: Travelling waves for the Gross-Pitaevskii equation. II. Comm. Math. Phys. 285(2), 567-651 (2009)

8. Bethuel, F., Saut, J.-C.: Travelling waves for the Gross-Pitaevskii equation. I. Ann. Inst. H. Poincaré Phys. Théor. 70(2), 147-238 (1999)

9. Bona, J., Ponce, G., Saut, J.-C., Sparber, C.: Dispersive blow-up for nonlinear Schrödinger equations revisited. J. Math. Pures Appl. (9) 102(4), 782-811 (2014)

10. Bony, J.-M.: Calcul symbolique et propagation des singularités pour les équations aux dérivées partielles non linéaires. Ann. Sci. École Norm. Sup. (4) 14(2), 209-246 (1981) 
11. Carles, R., Danchin, R., Saut, J.-C.: Madelung. Gross-Pitaevskii and Korteweg. Nonlinearity 25(10), 2843-2873 (2012)

12. Cazenave, T.: Semilinear Schrödinger equations, volume 10 of Courant Lecture Notes in Mathematics. New York University Courant Institute of Mathematical Sciences, New York (2003)

13. Chemin, J.-Y., Lerner, N.: Flot de champs de vecteurs non lipschitziens et équations de Navier-Stokes. J. Differ. Equ. 121(2), 314-328 (1995)

14. Coifman, R.R., Meyer, Y.: Au delà des opérateurs pseudo-différentiels, volume 57 of Astérisque. Société Mathématique de France, Paris, 1978. With an English summary

15. Colliander, J., Keel, M., Staffilani, G., Takaoka, H., Tao, T.: Global well-posedness and scattering for the energy-critical nonlinear Schrödinger equation in $\mathbb{R}^{3}$. Ann. Math. (2) 167(3), 767-865 (2008)

16. de Laire, André: Non-existence for travelling waves with small energy for the Gross-Pitaevskii equation in dimension $N \geq 3$. C. R. Math. Acad. Sci. Paris 347(7-8), 375-380 (2009)

17. Gallo, C.: Schrödinger group on Zhidkov spaces. Adv. Differ. Equ. 9(5-6), 509-538 (2004)

18. Gérard, P.: The Cauchy problem for the Gross-Pitaevskii equation. Ann. Inst. H. Poincaré Anal. Non Linéaire 23(5), 765-779 (2006)

19. Germain, P., Masmoudi, N., Shatah, J.: Global solutions for 3D quadratic Schrödinger equations. Int. Math. Res. Not. IMRN 3, 414-432 (2009)

20. Germain, P., Masmoudi, N., Shatah, J.: Global solutions for the gravity water waves equation in dimension 3. Ann. Math. (2) 175(2), 691-754 (2012)

21. Germain, P., Masmoudi, N., Shatah, J.: Global existence for capillary water waves. Commun. Pure Appl. Math. 68(4), 625-687 (2015)

22. Ginibre, J., Hayashi, N.: Almost global existence of small solutions to quadratic nonlinear Schrödinger equations in three space dimensions. Math. Z. 219(1), 119-140 (1995)

23. Grafakos, L., Kalton, N.J.: The Marcinkiewicz multiplier condition for bilinear operators. Studia Math. 146(2), 115-156 (2001)

24. Gravejat, P.: A non-existence result for supersonic travelling waves in the Gross-Pitaevskii equation. Commun. Math. Phys. 243(1), 93-103 (2003)

25. Gravejat, P.: Asymptotics for the travelling waves in the Gross-Pitaevskii equation. Asymptot. Anal. 45(3-4), 227-299 (2005)

26. Guo, Y., Pausader, B.: Global smooth ion dynamics in the Euler-Poisson system. Commun. Math. Phys. 303(1), 89-125 (2011)

27. Gustafson, S., Nakanishi, K., Tsai, T.-P.: Scattering for the Gross-Pitaevskii equation. Math. Res. Lett. 13(2-3), 273-285 (2006)

28. Gustafson, S., Nakanishi, K., Tsai, T.-P.: Global dispersive solutions for the Gross-Pitaevskii equation in two and three dimensions. Ann. Henri Poincaré 8(7), 1303-1331 (2007)

29. Gustafson, S., Nakanishi, K., Tsai, T.-P.: Scattering theory for the Gross-Pitaevskii equation in three dimensions. Commun. Contemp. Math. 11(4), 657-707 (2009)

30. Hayashi, N., Mizumachi, T., Naumkin, P.I.: Time decay of small solutions to quadratic nonlinear Schrödinger equations in 3D. Differ. Integral Equ. 16(2), 159-179 (2003)

31. Hayashi, N., Naumkin, P.I.: On the quadratic nonlinear Schrödinger equation in three space dimensions. Int. Math. Res. Not. 3, 115-132 (2000)

32. Jones, C.A., Roberts, P.H.: Motions in a bose condensate. iv. axisymmetric solitary waves. J. Phys. A Math. Gen. 15(8), 2599 (1982)

33. Jones, C.A., Putterman, S.J., Roberts, P.H.: Motions in a bose condensate. v. stability of solitary wave solutions of non-linear schrodinger equations in two and three dimensions. J. Phys. A Math. Gen. 19(15), 2991-3011 (1982)

34. Kawahara, Yuichiro: Global existence and asymptotic behavior of small solutions to nonlinear Schrödinger equations in 3D. Differ. Integral Equ. 18(2), 169-194 (2005)

35. Killip, R., Tadahiro, O., Pocovnicu, O., Vişan, M.: Global well-posedness of the Gross-Pitaevskii and cubic-quintic nonlinear Schrödinger equations with non-vanishing boundary conditions. Math. Res. Lett. 19(5), 969-986 (2012)

36. Mariş, M.: Traveling waves for nonlinear Schrödinger equations with nonzero conditions at infinity. Ann. Math. (2) 178(1), 107-182 (2013)

37. Ryckman, E., Visan, M.: Global well-posedness and scattering for the defocusing energy-critical nonlinear Schrödinger equation in $\mathbb{R}^{1+4}$. Am. J. Math. 129(1), 1-60 (2007)

38. Strauss, W.: Nonlinear scattering theory at low energy. J. Funct. Anal. 41, 110-133 (1981) 